\title{
Developments in turbulence research: a review based on the 1999 Programme of the Isaac Newton Institute, Cambridge
}

\author{
By J. C. R. HUNT $T^{1} \dagger$, N. D. SA N DHA M ${ }^{2}$, \\ J. C. VASSILICOS ${ }^{1}+$, B. E. LAUNDER ${ }^{4}$, \\ P. A. MONKEWITZ ${ }^{3}$ AND G. F. HEWITT ${ }^{5}$ \\ ${ }^{1}$ Department of Applied Mathematics and Theoretical Physics, University of Cambridge, \\ Silver Street, Cambridge CB3 9EW, UK \\ ${ }^{2}$ Aeronautics and Astronautics, School of Engineering Sciences, University of Southampton, \\ Southampton SO17 1BJ, UK \\ ${ }^{3}$ Institut de Machines Hydrauliques et de Mécanique des Fluides, Ecole Polytechnique Fédérale de \\ Lausanne, CH-1015 Lausanne, Switzerland \\ ${ }^{4}$ Department of Mechanical Engineering, UMIST, PO Box 88, Manchester M60 1QD, UK \\ ${ }^{5}$ Department of Chemical Engineering and Chemical Technology, Imperial College, Prince \\ Consort Road, London SW7 2BY, UK
}

(Received 1 February 2000 and in revised form 6 November 2000)

Recent research is making progress in framing more precisely the basic dynamical and statistical questions about turbulence and in answering them. It is helping both to define the likely limits to current methods for modelling industrial and environmental turbulent flows, and to suggest new approaches to overcome these limitations. Our selective review is based on the themes and new results that emerged from more than 300 presentations during the Programme held in 1999 at the Isaac Newton Institute, Cambridge, UK, and on research reported elsewhere. A general conclusion is that, although turbulence is not a universal state of nature, there are certain statistical measures and kinematic features of the small-scale flow field that occur in most turbulent flows, while the large-scale eddy motions have qualitative similarities within particular types of turbulence defined by the mean flow, initial or boundary conditions, and in some cases, the range of Reynolds numbers involved. The forced transition to turbulence of laminar flows caused by strong external disturbances was shown to be highly dependent on their amplitude, location, and the type of flow. Global and elliptical instabilities explain much of the three-dimensional and sudden nature of the transition phenomena. A review of experimental results shows how the structure of turbulence, especially in shear flows, continues to change as the Reynolds number of the turbulence increases well above about $10^{4}$ in ways that current numerical simulations cannot reproduce. Studies of the dynamics of small eddy structures and their mutual interactions indicate that there is a set of characteristic mechanisms in which vortices develop (vortex stretching, roll-up of instability sheets, formation of vortex tubes) and another set in which they break up (through instabilities and selfdestructive interactions). Numerical simulations and theoretical arguments suggest that these often occur sequentially in randomly occurring cycles. The factors that

$\dagger$ Now at Departments of Space and Climate Physics and Geological Sciences, University College, Gower Street, London WC1H 0AH, UK.

$\mp$ Present address: Department of Aeronautics, Imperial College, Prince Consort Road, South Kensington, London SW7 2BY, UK 
determine the overall spectrum of turbulence were reviewed. For a narrow distribution of eddy scales, the form of the spectrum can be defined by characteristic forms of individual eddies. However, if the distribution covers a wide range of scales (as in elongated eddies in the 'wall' layer of turbulent boundary layers), they collectively determine the spectra (as assumed in classical theory). Mathematical analyses of the Navier-Stokes and Euler equations applied to eddy structures lead to certain limits being defined regarding the tendencies of the vorticity field to become infinitely large locally. Approximate solutions for eigen modes and Fourier components reveal striking features of the temporal, near-wall structure such as bursting, and of the very elongated, spatial spectra of sheared inhomogeneous turbulence; but other kinds of eddy concepts are needed in less structured parts of the turbulence. Renormalized perturbation methods can now calculate consistently, and in good agreement with experiment, the evolution of second- and third-order spectra of homogeneous and isotropic turbulence. The fact that these calculations do not explicitly include highorder moments and extreme events, suggests that they may play a minor role in the basic dynamics. New methods of approximate numerical simulations of the larger scales of turbulence or 'very large eddy simulation' (VLES) based on using statistical models for the smaller scales (as is common in meteorological modelling) enable some turbulent flows with a non-local and non-equilibrium structure, such as impinging or convective flows, to be calculated more efficiently than by using large eddy simulation (LES), and more accurately than by using 'engineering' models for statistics at a single point. Generally it is shown that where the turbulence in a fluid volume is changing rapidly and is very inhomogeneous there are flows where even the most complex 'engineering' Reynolds stress transport models are only satisfactory with some special adaptation; this may entail the use of transport equations for the third moments or non-universal modelling methods designed explicitly for particular types of flow. LES methods may also need flow-specific corrections for accurate modelling of different types of very high Reynolds number turbulent flow including those near rigid surfaces.

This paper is dedicated to the memory of George Batchelor who was the inspiration of so much research in turbulence and who died on 30th March 2000. These results were presented at the last fluid mechanics seminar in DAMTP Cambridge that he attended in November 1999.

\section{Introduction}

'The problem of turbulence' has been seen as one of the great challenges of mathematics, physics and engineering for more than 100 years, by Lamb, Einstein, Sommerfeld, Ishlinski and others. Much of the interest in meeting this challenge is because of its practical value; the solution of many technical, industrial and environmental problems increasingly requires improvements, both in our fundamental understanding of turbulence, and in the utilization of advances in computation to calculate, at appropriate levels of accuracy and speed, the characteristic features and statistical properties of these flows (e.g. Hunt 1995; Holmes, Lumley \& Berkooz 1996).

Major centres for mathematical science and theoretical physics are holding intensive programmes on turbulence (examples being at Ascona, Monte Verita 2nd Symposium on Turbulence, Switzerland (Gyr, Kinzelbach \& Tsinober 1999) and the Institute for Theoretical Physics Santa Barbara in 2000) to complement regular summer schools and conferences, such as the European Turbulence Conferences (Frisch 1998) and Turbulent Shear Flow Symposia. In this paper we draw some general 
conclusions about current questions and developments in research on turbulence and its practical applications, resulting from the programme at the Isaac Newton Institute at Cambridge (UK) between January and June 1999. This involved more than 400 participants, visiting for various periods, and about 300 presentations by academic and governmental researchers, and those working on problems in industrial and environmental organisations, some of which combined with the Royal Academy of Engineering to provide generous support for the programme. All three disciplines of mathematics, physics and engineering were well represented. We also refer here to other recent research developments reported in the scientific literature and at the International Congress on Industrial and Applied Mathematics held at Edinburgh in July 1999. Detailed reports on various aspects of the programme have been or are being published by Voke, Sandham \& Kleiser (1999); Launder \& Sandham (2001); Vassilicos (2000b); Hunt \& Vassilicos (2000).

This report is aimed at a broad fluid mechanical readership. It focuses, inevitably somewhat selectively and subjectively, on progress in research towards the major questions of the subject and certain practical objectives, both of which provided a framework for the programme. Although these were formulated well before the programme began, they evolved by progressive adjustment and addition during the six-month period. They essentially finally became the following.

(i) To consider broadly and in depth whether fluid turbulence in its different manifestations has some common features (in some defined statistical sense) that are universal to all kinds of fully turbulent flow, or whether any commonality only exists within certain types of turbulence (such as those driven by mean shear, or natural convection). In other words is there one 'problem of turbulence' or several?

(ii) To explore the promising directions for tackling the fundamental problems of turbulence dynamics, some of which go back to the 1930s (see Constantin 2000; Frisch 1995). Within this fell the following specific questions.

(a) Is Taylor's (1938) conjecture about turbulence correct? It is that the normalized mean rate of energy dissipation, $\hat{\varepsilon}=\varepsilon /\left(u_{0}^{3} / L_{x}\right)$ (where $\varepsilon$ is the dimensional dissipation rate, $u_{0}$ is a typical r.m.s. velocity, and $L_{x}$ is a typical integral length scale) of a turbulent flow field (away from a boundary) is independent of the turbulent Reynolds number $R e=u_{0} L_{x} / v$, if the Reynolds number is sufficiently large, i.e.

$$
\hat{\varepsilon} \rightarrow \text { const } \quad \text { as } \quad R e \rightarrow \infty .
$$

If this is true (as is generally assumed in statistical models), what are the implications for the structure of the velocity field? If it is not, as some investigations suggest, what is the asymptotic relation between the rate of energy dissipation and the Reynolds number?

(b) What is the nature of the 'wiggliness' and 'smoothness' of the velocity field as $R e \rightarrow \infty$, a question first raised by Richardson (1926) who wondered whether the velocity, even though its magnitude is finite, might be so 'wiggly' that it is not effectively differentiable anywhere (as with a Weierstrass function or some other fields with a non-integral Hausdorff fractal dimension). An alternative concept is that as $R e \rightarrow \infty$, turbulence is fundamentally intermittent with a finite number of distinct points where the derivatives are singular, separated by smooth regions in between? Some combinations of such distributions of near-singularities (defined as singularities in the limit as the Reynolds number tends to infinity) are necessary if equation (1) is to be valid. Furthermore, how are such distributions consistent with the idea that velocity fields at the small scales may be self-similar over an increasing range of 
length scales as Re increases? How can deviations from self-similarity be considered in the context of multiple-scale velocity fields?

(c) Can even stronger singularities occur in which the velocity and vorticity at points in the flow tend to infinitely large values in a finite time $t^{*}$, after a finiteamplitude turbulent flow field has been initiated at $t=0$ ? Although this phenomenon has never been observed, some special mathematical solutions to the Euler and the Navier-Stokes equations suggest that it may be possible (Leray 1935; Kerr*, Moffatt*, Ohkitani \& Gibbon 2000; Doering \& Gibbon 2000). Are near-singularities of Navier-Stokes turbulence the remnants of finite-time singularities of the Euler equations? Does the tendency for such singular events to occur determine the 'tail' of the probability distribution of the turbulent flows and if so, how?

(d) What is the nature of the eddy transfer or 'cascade' process, in which when $R e \gg 1$ (if (1) is correct) the velocity fluctuations right down to the smallest scales reach a quasi-equilibrium state in the 'Lagrangian' or 'turn-over' time scale of order $L_{x} / u_{0}$ ? Also, to what extent are small-scale processes (depending on the precise definition) independent of the large-scale motions? Some physical models have suggested an infinite cascade involving vortical events at each 'eddy' scale (Tennekes \& Lumley 1971; Frisch 1995), whereas others have suggested that relatively few complex events are needed (e.g. Lundgren 1982). The upscale energy transfer equally needs better understanding through study of the large-scale dynamics, which depends on how these eddy motions are correlated over large distances (see for example Monin \& Yaglom 1975).

(e) To what extent do the large-scale motions of the turbulence tend to become independent of initial and boundary conditions, or, if the flow was initially laminar, of the particular process of transition to turbulence (George*): is this by means of internal self-organization or by chaotic interactions or both? Landau \& Lifshitz (1959): "We have seen that, whatever the initial phases $\beta_{j}$, over a sufficiently long interval of time the fluid passes through states arbitrarily close to any given state, defined by any possible choice of simultaneous values of the phase $\varnothing_{j}$. Hence it follows that, in the consideration of turbulent flow, the actual initial conditions cease to have any effect after sufficiently long intervals of time. This shows that the theory of turbulent flows must be a statistical theory." Batchelor (1953)'s view was more conditional: ".. we put our faith in the tendency for dynamical systems with a large number of degrees of freedom, and with coupling between these degrees of freedom, to approach a statistical state which is independent (partially, if not wholly) of the initial conditions. With this general property of dynamical systems in mind, rather than investigate the motion consequent upon a particular set of initial conditions, we explore the existence of solutions which are asymptotic in the sense that the further passage of time changes them in some simple way only." This and the other fundamental questions, provide a context for considering the appropriate future directions for the statistical computational models of turbulence needed for practical purposes.

(f) How are fully developed turbulent velocity fields related to their sources of energy whether from initial conditions, continuing instabilities within a flow, or from boundary conditions such as a rigid wall?

(iii) To consider changes in ideas about the fundamental statistical properties of turbulence. Whereas the previous questions have only slightly changed over the past 50 years, those about the fundamental statistical properties of turbulence, although they are based on earlier theories, have changed more significantly as more detailed measurements have been made. The first three listed below are based on Kolmogorov's 
(1941, 1962) theories and their extensions and have been reviewed by Frisch (1995), and Sreenivasan \& Antonia (1998). The last two concern the basic concepts of inhomogeneous turbulence due to Prandtl (1925) and von Kármán (1930), which apply to a wide range of turbulent flows independently of initial conditions.

(a) How do the mean value of $n$ th-order moments $S_{n}$, where $S_{n}=\overline{\Delta u^{n}(r)}$, of the difference in velocity $\Delta u(r)$ between points separated by a distance $r$ vary with $n$ when $r$ is less than the integral scale $L_{x}$ and much greater than the viscous microscale $\eta$ ? Is there a universal result when $R e \rightarrow \infty$ for $S_{n}$ normalized on the mean (or some conditional mean) value of $\varepsilon, \bar{\varepsilon}$ of the form

$$
S_{n}(r)=C_{n} \bar{\varepsilon}^{n / 3} r^{n / 3} \text { for } n>1, \eta \ll r \ll L_{x} .
$$

If not, is there some less general power law such as

$$
S_{n}(r) \propto r^{\zeta(n)},
$$

and if so, what determines $\zeta(n)$ ? What is the nature of the dependence of $C_{n}$ or $\zeta(n)$ on the large scales, which in general are non-isotropic and inhomogeneous? How is this dependence related to the recently observed anisotropy of the structure functions (Noullez et al. 1997; van de Water, Staicu \& Guegan 2000)? Are these small-scale statistics affected by particular characteristics such as the mean shear?

(b) Do the Lagrangian $n$ th-order moments $S_{n}^{(L)}$ of the velocity $\Delta u(\Delta t)$ of a fluid particle at two points separated in time by $\Delta t$ scale similarly on $\bar{\varepsilon}$ ? For example (as a generalization of Inoue 1951) does

$$
S_{n}^{(L)}(\Delta t)=C_{n}^{(L)} \bar{\varepsilon}^{n / 2}(\Delta t)^{n / 2} .
$$

Can the same scaling apply to the related moment of the separation distance $\Delta(t)$ between marked fluid particles released at time $t=0$, such that

$$
\overline{[\Delta(t)]^{n}}=C_{\Delta} \bar{\varepsilon}^{n / 2} t^{3 n / 2} .
$$

(c) What are the fundamental statistical distributions governing the observed intermittency of the smallest-scale gradients and dissipation rate $\varepsilon$ ? The form of the probability distribution of $\varepsilon$ affects the quantitative value of $\zeta(n)$ in $(2 b)$ (Kolmogorov 1962), but so also does the nature of near-singularities in the turbulence.

(d) Are certain statistical properties of fully developed inhomogeneous turbulence near plane rigid surfaces independent of the upstream or outer flow conditions and what is their form? This question refers to flows with and without a significant velocity $\bar{U}$ greater than the typical fluctuating velocity $u_{*}$; firstly, what is the mean velocity profile $\bar{U}\left(x_{3} u_{*} / v\right)$, whose mathematical form may be determined by the dependence on the Reynolds number of the outer flow (Barenblatt \& Chorin 1998)? Secondly under what conditions are the velocity spectra $\Phi_{11}\left(k_{1}\right)$ and $\Phi_{22}\left(k_{1}\right)$ along the streamwise direction given by

$$
\Phi_{11}\left(k_{1}\right), \Phi_{22}\left(k_{1}\right) \propto u_{*}^{2} k_{1}^{-1},
$$

when $\Lambda^{-1}<k_{1}<x_{3}^{-1}$, for $x_{3} \ll h$, where $h$ is the thickness of the boundary layer/pipe and $\Lambda$ is an outer length scale much greater than $h$ (Marušic \& Perry 1995)? Thirdly, for turbulent flows with or without a mean velocity component, how general is the self-similar form of the two-point velocity correlation of the normal components

$$
R_{33}\left(x_{3}, x_{3}^{\prime}\right)=\overline{u_{3}\left(x_{3}\right) u_{3}\left(x_{3}^{\prime}\right)} / \overline{u_{3}^{2}}\left(x_{3}\right)=f\left(x_{3} / x_{3}^{\prime}\right) \text { for } x_{3}<x_{3}^{\prime}
$$

(Hunt et al. 1989)?

(e) To what extent do the asymptotic forms as $R e \rightarrow \infty$ for the statistics and 
characteristic eddy structures differ from those found when $R e$ is finite? Are there distinct subclasses of turbulence corresponding to different ranges of $R e$ (or of Rayleigh number for natural convection (cf. Castaing et al. 1989))?

(iv) To consider how fundamental research on turbulence might lead to improvements in turbulence-simulation methods and statistical models. The deficiencies of current models, as pointed out by industrial participants, tend to become apparent when they are applied to turbulent flows that are highly inhomogeneous and rapidly changing (over the length and time scales of the large eddies), which is to be expected since these 'non-conforming' situations do not correspond with the assumptions that underpin the models, e.g. Launder \& Spalding (1972), Lumley (1978). Because industry is now familiar with the use of such models, it was requested that their rationale and limitations should be defined and explained using recent research, such as that on inhomogeneous turbulence. Since the models are often applied to 'non-conforming' flows, interest was expressed in interpreting the often puzzling results of the computations in these situations. Moreover, significant modifications are being proposed to existing modelling methods and these need to be evaluated and understood.

Questions (reviewed by Geurts*; see also Geurts \& Leonard 1999) about the limitations of large-eddy simulation methods are closely linked to those on the fundamental dynamics and statistics, since the methods involve computing the 'resolved' velocity field above a certain 'filter' scale $l_{f}$ that is greater than that of the smallest 'Kolmogorov' eddies of the turbulence $l_{K}$. (Only if the Reynolds number of the turbulence is small enough, typically $R e<10^{3}$, is it possible to avoid this approximation and compute the turbulence directly, e.g. Moin \& Mahesh 1999). Discussions were mainly focused on constant-density flows, though the importance of turbulence in two-phase flows (Hewitt*; Reeks*), buoyancy-dominated flows (Banerjee*; Launder*), and compressible flows (Bonnet*; Gatski*) were reviewed. There are many detailed questions about this filtering approximation; for example what happens when very small-scale, highly anisotropic and often non-Gaussian motions are generated near boundaries, or how is the predictability of a simulated flow affected by randomness of the unresolved small scale, a problem of interest for forecasting environmental flows and controlling engineering flows (Lesieur 1999).

\section{Origins}

Turbulent flows are generated in different ways. Laminar flows (i.e. flows that in any one realization in a fluid with simple boundaries are exactly predictable for all time given a finite amount of data about the flow) can become unstable when small fluctuations develop nonlinearly. Turbulent flows can also originate from fluctuations caused by boundaries with complex (fractal) shapes (Queiros-Conde \& Vassilicos 2000) and complex movements (Warhaft*) or by the effects of body forces, e.g. electro-magnetic forces. A fully developed turbulence is reached when one or more of these processes has generated velocity fields that are chaotic in space and time, having smooth spectra and smooth probability distributions. Once this state is reached, which requires that the Reynolds number is large enough, these general qualitative properties are observed not to change even when quite substantial perturbations are introduced, say in relation to $u_{0}$ and $L_{x}$, such as meanflow distortions or damping via body forces or suspended particles. In flows with certain interactive body forces (e.g. electromagnetic or gravitational) the turbulence can generate resonances with local singularities (Kerr*; McGrath, Fernando \& Hunt 1997). A significant mechanism, discussed later, for increasing the level of chaotic 
behaviour in turbulence at high values of $R e$ is the continuous growth of instabilities from infinitesimal initial amplitudes even when the turbulence is fully developed.

A key question of recent research has been to identify and describe the different mechanisms affecting the evolution of unstable fluctuations from perturbations on laminar flow into fully developed turbulence. The first of two types of transition proposed by C. C. Lin (Ffowcs Williams, Rosenblat \& Stuart 1969) was a 'slow' evolution (as in wakes, jets and curved shear flows) when the initial shear flow is unstable to a single mode and there are distinct bifurcations as subsequent modes develop by nonlinear interaction. The second type is a fast evolution (as in pipe flow) when nonlinear growth of a single transition rapidly produces a spectrum of velocity fluctuations comparable in width to that of fully developed turbulence. In this case there is a 'fast' transition or breakdown and a sudden change from smooth laminar flow to turbulence or patches of turbulence. These concepts are consistent with the mathematical demonstration of Ruelle \& Takens (1971) that (whether the transition is slow or fast) a smooth spectrum with finite width can exist at finite $R e$.

These categories can be understood physically by considering how these instabilities develop in particular flows. For example, in boundary layers, disturbances grow in the streamwise direction via a 'slow' transition process with distinct modes, typically evolving from linear to three-dimensional weakly nonlinear form (Smith*). Depending on whether the streamwise extent of the boundary layer flow is short or long, the disturbances may or may not develop into turbulence for a given value of $R e$. In other flows, for example with recirculation, such as Taylor-Couette or certain wake flows, the disturbances stay at the same amplitude everywhere in the flow and do not necessarily generate turbulence, so that the slow evolution of nonlinearities implies slow transition. As Huerre \& Monkewitz (1990) first clarified, in the former case of the boundary layer the 'slowly' evolving instabilities are 'convective', and may or may not lead to transition anywhere in the flow. In the latter type of flow absolute instabilities fill the domain, and are slowly evolving. In most cases where there is a fast evolution of nonlinear disturbances, whether the instabilities are convective or absolute, there is fast transition to turbulence.

Recent research has provided some insights into the questions raised by this framework, but the framework itself has not seriously been questioned. Whereas many features of slow instabilities have been analysed, the key fundamental question is to understand the breakdown process, which can also reappear in a fully turbulent flow as a 'transition' from one form of turbulence structure to another - see below. The most thoroughly studied case is that of global instability of rotational flows with locally closed elliptical streamlines (and particle paths), whose general significance for turbulence was pointed out by Gledzer et al. (1975), Malkus \& Waleffe (1991) and others, and whose theoretical understanding was developed by Bayly (1986). The discussions showed that both global (referring to a non-local classical linear analysis in terms of normal modes) and local (referring to WKB short-wave asymptotics along individual trajectories - see Lifshitz \& Hameiri 1991) solutions along streamlines (see Cambon \& Scott 1999; Leonard*) lead to the exponential growth of three-dimensional, wave-like disturbances. Disturbances grow over times that scale on the inverse of the strain rate and not on the inverse of vorticity. These two time scales can be comparable but they can also be very different as in the vortex interaction experiments of Leweke \& Williamson (1998). Nonlinear interactions develop over a period of the order of the rotation time (and not significantly less), leading to a fully developed, multi-lengthscale, turbulent flow, i.e. a breakdown. Recent laboratory experiments on a laminar vortex distorted by an adjacent vortex by Leweke \& Williamson (1998) and on a fully 
turbulent vortex undergoing compression by Borée et al. (1999) demonstrate how this basic inviscid instability (Lundgren \& Mansour 1997) causes rapid transition of laminar flows and a rapid change in the structure of a turbulent flow. This is consistent with the integral of helicity, $H=\int \boldsymbol{u} \cdot \omega \mathrm{d} V$, being conserved for these isolated structures or contained flows. Note that the local helicity $h=\boldsymbol{u} \cdot \boldsymbol{\omega}$ increases from zero at the base flow to positive and negative values of order $u_{0}^{2} / L_{x}$, showing that increasing $|h|$ is not necessarily an indicator of a slower cascade of vorticity.

With an analysis employing global instability methods, Le Dizes* showed that the mechanisms involved in the elliptic instability are essentially equivalent to the growth of three-dimensional perturbations within vortices explained as 'vortex core dynamics' by Hussain*.

New qualitative evidence presented by Durbin* from the numerical simulations of Wu et al. (1999) was consistent with the analysis of Malkus \& Waleffe (1991), in that these global or elliptical instabilities are the cause of the rapid evolution of boundary layer instabilities when the imposed disturbances (whether at the wall or from external velocity fluctuations) have a significant amplitude $u_{e}$. These produce sufficiently large closed streamline regions around the critical layer for rapid three-dimensional instabilities to grow locally in the form of the very low-amplitude disturbances. 'Bottom up' (i.e. forward pointing) triangular spots are generated at the wall while 'top down' (backward pointing) spots are generally formed from very low amplitude disturbances imposed at the top of the boundary layer. Typically, the former rapid evolution and transition occurs if $u_{e}$ is comparable to the fluctuations in the fully turbulent boundary layer, i.e. $u_{e} \sim u_{*}$ where $u_{*}$ is the friction velocity, typically $U_{0} / 20$ where $U_{0}$ is the mean velocity outside the layer.

A qualitative understanding of this spot transition is currently applied in the design of turbo-machinery blades. As it passes downstream, the entrainment flow into the growing spot affects the mean velocity profile, so as to reduce the deficit in the momentum flux of the boundary layer profile and its tendency to thicken in the adverse pressure gradient towards the trailing edge of the aerofoils (Hodson*).

The effects of different kinds of external turbulence on the transition to turbulence of laminar boundary layers on isolated bodies can only be analysed theoretically in the initial stages of transition for the idealized case of a very thin flat plate and when the amplitude of external fluctuations have a vanishingly small amplitude (e.g. Wundrow \& Goldstein 1999). For practical situations, various approximate theoretical methods have been developed for different types of laminar flow and external turbulence (Atkin*). Some concepts were presented about how velocity fluctuations of free-stream turbulence outside a boundary layer affect fluctuations within it and cause 'bypass' of the transition process via a sequence of instabilities. The first mechanism is that fluctuations are 'convected' along the streamlines and enter the growing boundary layer where its velocity gradient amplifies the small-scale fluctuations algebraically (Voke*; see also Trefethen et al. 1993) and where largerscale fluctuations may amplify the eigenmodes of the layer. Different methods for calculating the amplification of disturbances in shear flows where the streamlines are slightly non-parallel were discussed (Lingwood*), in particular the relative merits of the widely used parabolized stability equations (PSE) and the rigorous triple-deck asymptotic theory (Lucchini*; Healey*). The second mechanism is caused by the action of external fluctuations travelling over the layer as a localized disturbance, such as a moving wake. Numerical simulations confirm the theory that if they travel at the same speed as the free-stream speed $U_{0}$, the fluctuations they induce tend to be maximal at the top of the layer because the flow within the layer is 'sheltered' from the 
larger scales of the external turbulence; the smaller scales can induce fluctuations and diffuse downwards. However the upper fluctuations rapidly grow as Kelvin-Helmholtz billows and spread the resulting small-scale turbulence downwards. Both effects can cause transition. In engineering models they are usually represented as a diffusion-like transport process in one-point statistical models (Savill*). Durbin* argued that such approximations might be incorrect because they imply that the external turbulence would have a greater effect on the boundary layer when its length scale is increased.

The third major area of controversy about the initiation and persistence of turbulence concerns the cycle of growth and decay of fluctuations in turbulent boundary layers near a rigid surface (or 'wall'). There are minor differences between the mechanisms proposed for the lower range of the turbulent Reynolds number $(R e)$, but a major difference in the proposed self-generation mechanisms when $R e$ becomes very large. When $R e \lesssim 10^{4}$, as many numerical simulation and laboratory measurements have demonstrated, instabilities in initially laminar boundary layers become nonlinear, and then develop into longitudinal vortices. These deflect upwards and downwards the mean spanwise vorticity of the boundary layer, causing low-speed and higherspeed strips with associated elongated vorticity fields. These become unstable, grow, and disrupt the local flow structure; significant velocity fluctuations are generated that may extend into the outer layer; following their decay, the streamwise vortical regions re-form once more (Jimenez*; Sandham*; Hussain*). In order to describe the possible mechanisms, elements of the flow field have been studied in isolation, e.g. by conditional sampling (Hussain*), or by proper orthogonal decomposition of the measured two-point velocity correlations (Holmes et al. 1996). Different techniques have then been used to analyse these fields, for example by local stability analysis or by calculating the temporal evolution of a few low-order modes.

While the main picture, as described above, is common to all investigations, there are some significant differences among the models, particularly regarding whether the near-wall dynamics is a pure instability mechanism quite independent of the fluctuation velocity field in the outer part of the boundary layer (or channel flow) (Hussain*), or whether random fluctuations in this outer field stimulate resonant modes near the wall. This interaction may be essential to sustain the cycle of growth and decay of the 'near wall' vortical structures (Sandham*). Apparently no experimental evidence yet exists that leads to a clear distinction between the validity of these concepts.

In turbulent boundary layers the continued generation of small-scale instabilities or resonances near the wall at moderate $R e$ means that the flow is susceptible to being controlled, for example by adjusting small panels up and down to modify the growth of instabilities (Hussain*; Holmes et al. 1996; Carpenter*). If the eddy structure undergoes substantial change at very high Reynolds number, as indicated by experiments, will the effectiveness of such wall techniques be reduced? Another turbulence-control technique is the introduction of long-chain molecules which reduce the frictional drag of liquid flows in large pipelines; since the mechanism here is through 'damping' of eddy straining, it should not change qualitatively at high Reynolds number, as is observed (Sreenivasan*).

In most turbulent free shear flows, the effect of instabilities on the mean flow and turbulence is surprisingly not generally considered to be so significant, because they occur on the edges of the free shear layer. In these flows the energy of the turbulence is mostly generated in the interior of the flow where there is a strong local interaction between the gradient of the mean velocity $\nabla U$ and the Reynolds stresses $\overline{u_{i} u_{j}}$. The role of instabilities may be particularly significant on the edges of clouds and plumes when influenced by body forces and external turbulence (e.g. Baht \& Narasimha 1996). 


\section{New measurements and simulations}

The most universal and fundamental aspects of turbulence, namely the small-scale statistical structure when $R e$ is large enough for a significant inertial, spectral subrange to exist, can still only be studied in detail through measurements, because numerical simulations and theoretical models are only approximate. Furthermore, no facilities yet exist in which it is possible to mount controlled experiments with a well-designed velocity field at very high Reynolds numbers (Nieuwstadt*); the measurements still have to be made in artificial turbulent flows constructed for other purposes, such as in one part of an aeronautical wind tunnel where $R e \sim 10^{4}-10^{5}$ (Arneodo et al. 1999; Saddoughi \& Veeravalli 1994), or in strong jets (Van Atta 1991), or else in the atmospheric boundary layer where $R e \gtrsim 10^{5}$. As explained in $\S 1$, many measurements in these flows of the $n$ th-moments $\overline{\Delta u^{n}(r)}$ of the velocity difference between two points separated by a distance $r$ have shown that for $n=2,3, \ldots$, the results approximately follow the predictions of $(2 a)$. The departures in the theoretical exponent of the higher-order structure functions from the predicted values were attributed to the fluctuations in the energy dissipation rate $\varepsilon$ following Kolmogorov's modified theory (1962) (recently reviewed by Sreenivasan \& Antonia 1998).

Recently, large wind tunnel and atmospheric measurements have related the smallscale motions to the velocity field as a whole, $u(x, t)$ for example by calculating the conditional $n$ th-order moments of components of $\Delta u(r)$ at each value of $|u|$, denoted by $\overline{\left(\Delta u^{n} ;|u|\right)}$ (Praskovsky et al. 1993; Tsinober*; Sreenivasan*). These results showed some dependence of the amplitude and even the structure of small-scale eddy motion on the large-scale eddy motion. In this context we note that Nie \& Tanveer (1999) rigorously derived, from the Navier-Stokes equation, Kolmogorov's four-fifths law for the third-order structure function (equation ( $2 a)$ for $n=3$ with $C_{3}=4 / 5$ ) without Kolmogorov's assumption of local isotropy. Hence, some elements of the small-scale structure can persist even in the presence of large-scale effects. We conclude that the mechanisms for direct connections between large and small scales in different types of turbulence will only be better understood with more detailed and different types of statistical measurements.

These new experimental and theoretical results are nevertheless consistent with earlier studies showing significant amplitudes of the non-isotropic component of the second- and higher-order moments of small-scale turbulence that were dependent on the large-scale mean shearing motion or the non-isotropic, non-Gaussian eddies of natural convection (Saddoughi \& Veeravalli 1994; Hunt, Kaimal \& Gaynor 1988). Other kinds of local conditional statistics taken effectively at two points were reported that were designed to elucidate the detailed structures of small-scale eddies at high $R e$. Wavelet analysis of these measurements is a natural generalization of the structure-function analysis of these measurements providing increased information both in physical and scale spaces (Arneodo et al. 1999; Brasseur*). For example, the wavelet transform was applied to the study of the dynamics of the Burgers equation leading to a clear demonstration of how a single localized characteristic flow structure in the field (in this case in the form of a shock) can all by itself determine the highwavenumber energy spectrum, and how energy transfer can be studied concurrently in both scale and physical spaces (Brasseur*). Furthermore, by applying wavelet methods to experimental, one-point turbulence velocity data, Arneodo et al. (1999) were able to show that if the turbulence is a multiplicative cascading process (which it may well not be), then this process is not self-similar. From their atmospheric measurements of low-order and higher statistics, Kholmyansky \& Tsinober* and Sreenivasan* deduced that small-scale turbulence may not be completely self-similar 
at the Reynolds numbers currently attainable. However, flow visualization studies in the laboratory (Schwarz 1990; Douady, Couder \& Brachet 1991), casual observations in the environment of dust, bubbles, clouds etc., and numerical simulations at lower Re (Passot et al. 1995; Jiménez et al. 1993; Ohkitani*) have shown that characteristic structures exist in the form of rolled up vortical layers and elongated vortices. However, there is still no conditioned experimental data for these structures that is sufficiently detailed for their precise analysis.

Although flow visualization provides multi-point qualitative information this can only be provided systematically by making measurements at three or more points simultaneously. Warhaft* showed how three-point velocity and temperature measurements in active grid turbulence (which produces enough energy to simulate many features of high-Re turbulence) could demonstrate quite clearly the existence of 'scalar/vortical fronts' on thin surfaces across which there are intense scalar gradients (see Mydlarski \& Warhaft 1998; Chertkov, Pumir \& Shraiman 2000). This is consistent with numerical simulation and theoretical concepts about the first stage of intense scalar mixing, the second stage being the rolling up of these fronts leading to intense local mixing (Nieuwstadt*).

A complementary approach to understanding turbulence structure is to consider how the relative velocity $\Delta u(t)$ and the distance $\Delta$ between pairs of fluid particles vary with time $t$ (and the initial spacing $\Delta_{0}$ at $t=0$ ). Richardson's (1926) atmospheric measurements, that helped stimulate the Obukhov-Kolmogorov theory, suggest that

$$
\overline{\Delta^{2}}=G_{\Delta} \varepsilon t^{3},
$$

when $\Delta$ is smaller than the turbulence length scale $L$. This statistical relation, which has great practical value for estimating concentration fluctuations (Derbyshire, Thomson \& Woods*), can now be understood better in terms of the eddy motions in the turbulence: an approach of practical value on the scale of synoptic storms. (A collaboration between industrialists and academics was initiated during the programme to use this approach for establishing the limitations of Richardson's law.) Laboratory experiments (albeit at quite low $R e \lesssim 10^{2}$ ) of Tabeling* using the new techniques of simultaneously measuring $\Delta$ and the pattern of the flow field (with particle imaging), showed how $\overline{\Delta^{2}}$ increases mostly because of sudden separation events between a minority of particle trajectories, the majority of them remaining close to each other for a very long time. This is consistent with simulation results and the approximate theory of Fung et al. (1992) who argued that these rare, sudden and intense separation events occur in saddle-point regions where streamlines converge and diverge most rapidly (where the 'scalar fronts' described above tend to form). It may be because of the scarcity of these particle separation bursts that, in some flow fields, the Richardson constant $G_{\Delta}$ in equation (7) is of the order 0.1 or smaller (see also $\S 4.2$ ). There remains much uncertainty about this fundamental constant and the generalization of (3) to high moments, that can only be settled by experiments at high enough $R e$ for there to be a wide self-similar 'inertial range' (cf. Voth, Satyanarayan \& Bodenschatz 1998).

Turbulent flows in practice are inhomogeneous and bounded either by a rigid wall or by a region of non-turbulent flow in which there might be some kind of laminar motion or none at all. In the latter case, there is a transition between the turbulent and laminar flow, with a randomly moving 'interface' separating the rotational velocity fluctuations of the turbulence from irrotational motion, which decay to zero over a distance $L_{x}$ from the interface. Recent experiments and simulations for both these types of boundary are more detailed than earlier studies and suggest that new concepts and models are necessary for these critical boundary regions of turbulent flows. 
Although the structure of turbulence near a 'wall' in the absence of mean flow has only been studied in detail over the past 20 years, its main features (for convective turbulence or mechanically generated turbulence) have now been established through similar findings in experiments, e.g. Kit, Strang \& Fernando (1997), numerical simulations, e.g. Perot \& Moin (1995a,b), Banerjee*, and approximate models, e.g. Craft \& Launder (1996): namely that the length scales of eddy motions parallel to the wall are largely determined by the length scales of eddy motion away from the wall $L_{x}$ (e.g. as in thermal convection, Castaing et al. 1989), while the length scales normal to the wall are determined by the distance $\left(x_{3}\right)$ to the wall. The normal velocity decreases towards the wall and parallel components increase (by up to $30 \%$ ) until they are within a fluctuating shear layer of thickness $\mathscr{L} \ll L_{x}$. For $R e$ below about $10^{3}$, the surface shear layer produced by the energy-containing eddies is laminar and its thickness is of order $R e^{-1 / 2}$. As $R e$ increases above about $10^{4}$, this overall structure, including the form of the spectra, does not change, but the shear layer at the wall changes character and its thickness $\mathscr{L}$ becomes approximately proportional to $(\log R e)^{-3}$ as opposed to $R e^{-1 / 2}$. For a smooth wall there is a very thin inner viscous layer of thickness $h_{*} \sim v / u_{*}$ where $u_{*}$ is the friction velocity of the energy-containing eddies. (See reviews in Plate et al. 1998.)

By contrast, when there is a mean shear flow parallel to the wall the eddy structure is quite different and has a greater qualitative change as $R e$ increases, especially in its relation to the eddy structure far above the wall. Measurements have been recently reported of the spectra $\Phi_{11}, \Phi_{22}\left(k_{1}\right)$ of the streamwise and spanwise velocity fluctuations in turbulent pipe flows of radius $h$ for $R e$ up to $10^{4}$ (Kim \& Adrian 1999) and in the atmospheric boundary layer of thickness $h$ for $\left(R e>10^{5}\right)$ (e.g. Hoxey \& Richards 1992; Fuehrer \& Friehe 1999). These confirmed over different ranges of $x_{3}$ the main result of the earlier studies of Marušic \& Perry (1995) and others; in pipes the range was close to the 'wall' (i.e. $h_{*}<x_{3}<h_{s} \sim 0.2 h$ ), while in the atmosphere the range was very close to the ground $\left(z_{0}<x_{3}<h_{s} \sim 0.01 h\right)$, where $h_{*} / h \sim 10^{-3}$ and $z_{0} / h \sim 10^{-4}$. In both cases the large-scale spectra had an invariant self-similar structure for eddy scales $\left(k_{1}^{-1}\right)$ greater than the distance $\left(x_{3}\right)$ from the wall, but less than a long streamwise length scale $(\Lambda)$ i.e. $(2 \pi / \Lambda) \geqslant k_{1} \geqslant\left(2 \pi / x_{3}\right)$. It was found that the spectra $\left(\Phi_{11}\left(k_{1}\right), \Phi_{22}\left(k_{1}\right)\right)=\left(C_{* 11}, C_{* 22}\right) u_{*}^{2} k_{1}^{-1}$, where $u_{*}$ is the surface friction velocity, and $C_{* 11}, C_{* 22}$ are approximately independent of $x_{3} / h$.

Not only is the depth $h_{s}$ of the surface layer, where this self-similar spectra is observed, sensitive to the value of $R e$, but so is the value of $\Lambda / h$. In both cases the maximum value of $\Lambda$ is significantly greater than both $h$ and the scale of the eddy structures in the outer part of the flow (which can be explained in terms of the formation of 'streaks' by vertical fluctuations interacting with the mean shear, Jimenez*). At lower $R e, \Lambda$ varies with $x_{3} / h$ quite rapidly in a pipe (Kim \& Adrian 1999 ) and less so in a boundary layer, but in both cases reaches about $18 h$ when $x_{3} / h \sim 0.2 h$, whereas in the very high $R e$ range of the atmospheric boundary layer $A$ is approximately equal to $3 h-5 h$ and does not vary significantly with $z$. (This formula, proposed by Davenport (1961), has been used by wind engineers ever since!) These experiments confirm the theoretical model of Townsend (1976) and Perry* that at very high $R e$ the variances of the parallel components $\overline{u_{1}^{2}}, \overline{u_{2}^{2}}$, obtained from integrating the spectra, vary in proportion to $u_{*}^{2}[\ln (\Lambda / z)+$ constant $]$. Most measurements now agree that in this range of wavenumber the spectra for the normal velocity component $\Phi_{33}\left(k_{1}\right)$ and the co-spectra of the shear stress $\Phi_{13}\left(k_{1}\right)$ are constant with wavenumber, i.e. $\Phi_{33}, \Phi_{13} \simeq\left(C_{* 33}, C_{* 13}\right) u_{*}^{2}$. So on integration these variances are proportional to $u_{*}^{2}$. This is consistent with the definition $u_{*}^{2}=-\overline{u_{1} u_{3}}$ and with the general result for high-Re shear flows that $\overline{u_{3}^{2}} /\left(-\overline{u_{1} u_{3}}\right.$ is of order unity. 
These statistical results are also broadly consistent with the main features of the eddy structure, namely the elongated contours of instantaneous high and low streamwise velocity, found in numerical simulation (Jimenez*) and atmospheric observations of elongated streamwise vortices, and with the sloping eddy structures expanding in diameter as the distance from the wall increases seen in laboratory experiments (Perry*).

The difference between the magnitude of $\Lambda / h$ and its variation with the normal distance $\left(x_{3} / h\right)$ as $R e$ increases much above $10^{4}$ is consistent with the possible change of the eddy structure. A more vigorous vertical exchange of large eddies from the outer region of boundary layers towards the wall is seen in the atmosphere in the form of moving 'cats paws' on water surfaces or cornfields, an increased value of the cross-correlation $\hat{R}_{33}\left(x_{3}, x_{3}\right)$, defined in (6) (Brown \& Thomas 1977; Hunt \& Morrison $2000)$, and an increase in the vertical turbulence $\left(u_{3}^{2}\right)$ with height $(z)$ in the surface layer (Högström 1990).

The turbulence in the outer regions of a boundary layer and throughout the whole thickness of free shear flows is dominated by the interface with the exterior nonturbulent flow. An industrial participant regretted that despite its importance for aeronautical applications this aspect of inhomogeneous turbulence has received far less research attention than that near the wall. As Lumley* remarked, the dynamics of these interfaces also determine how local regions of intense vortical motions evolve within a general turbulent flow when $R e$ is very large. Recent analysis by Bisset et al. (1998) of previously published numerical simulations of wakes (Moser, Rogers \& Ewing 1998), has shown that turbulence statistics have a local structure when expressed in terms of the normal distance $n_{I}$ from such interfaces. Even though the Reynolds number of these simulations were not large $\left(\operatorname{Re} \sim 10^{2}\right)$ it was found that the conditional profiles of the variables as a function of $n_{I}$ vary sharply near $n_{I}=0$ because of the very active small-scale motions at the interface; the vorticity variance $\overline{\omega^{2}}\left(n_{I}\right)$ and dissipation $\bar{\varepsilon}\left(n_{I}\right)$ were approximately constant for $n_{I} / h \lesssim-0.01$, and vanished for $n_{I} / h \gtrsim 0.01 h$, showing that the interface is even thinner than the expected scale of order $R e^{-3 / 4}$. Even the large-scale variables such as the conditional mean velocity $\bar{U}$ and temperature $\bar{T}$ also have sharp jumps at the interface. The computed flow fields show how large scales bring 'fresh' fluid from the interior of the region to the interface where it is mixed both at saddle-point regions (defined with respect to the moving surface) and at engulfing nodal regions at the back of the large folds in accordance with the experimental results of Ferre et al. (1990) and Gartshore (1966).

These results should help explain and improve some of the ad hoc steps taken in numerical calculations of interface processes which largely ignore the intermittency of the turbulence. The rate of boundary entrainment $E_{b}$ or slow movement outward of the mean interface position, i.e. $E_{b}=\mathrm{d} \overline{x_{3_{I}}} / \mathrm{d} t$ is approximated by a diffusion-like process in the models (Turner 1986). But since the eddy viscosity $v_{e}$ outside the interface is zero, this would mean $E_{b}=0$. Therefore, as explained by modellers (Leszchiner*), a small non-zero value of $v_{e}$ has to be assumed, although its magnitude has only a small effect on $E_{b}$ (cf. Cazalbou, Spalart \& Bradshaw 1994). Further studies are needed to resolve the uncertainty in the value of $E_{b}$ for turbulent layers, which experimenters and modellers find is of the order of $u_{*}$, the r.m.s. velocity fluctuation. George* on the other hand argued that $E_{b}$ is determined by a weak diffusive process and is much smaller, being of the order $u_{*}\left(u_{*} / U_{0}\right)$ (George \& Castillo 1994).

These discussions about the structure of the fluctuating velocity field at the wall and at the outer interface in turbulent boundary layers are related to the current 
controversies about the form of the mean velocity profile normalized on the surface friction velocity, $U\left(n_{*}\right) / u_{*}$, where $n_{*}=x_{3} u_{*} / v_{*}$. Since its discovery by von Kármán (1930) the profile has generally been accepted as having a logarithmic form, i.e.

$$
U / u_{*}=A \ln \left(n_{*}\right)+B,
$$

where $A \simeq 2.5$ and $B \simeq 5.6$ are experimental coefficients that were assumed to be effectively invariant with Reynolds number for $R e \gtrsim 10^{3}$. There is a similar log profile over rough surfaces. The form (8) is now questioned, firstly by close examination of new measurements in high-Re turbulent boundary layers (e.g. those of Zagarola \& Smits 1998), and secondly by reconsidering similarity theory, which leads Barenblatt \& Chorin (1998) to propose that the data are better described by a 'power law' profile of the form

$$
U / u_{*}=A^{\prime} n_{*}^{\alpha}+B^{\prime},
$$

where $\alpha$ is a function of $R e$. The largest differences between (8) and (9) (which are of the order of $10-20 \%$ ) appear at locations where $n^{*} \lesssim 10^{2}$. Perry* and other experimenters have commented that this is where the measurements are most uncertain because, at very high values of $R e$, the measurement points are so close to the wall that the accuracy of the measurement is not great enough to distinguish between the formulae. However these differences matter because even small changes of say $3 \%$ in the pressure drop along pipes or in the skin friction of aircraft are economically significant. Establishing the form of this universal near-wall profile is also considered essential as a boundary condition for many widely used statistical models applied to this kind of turbulent flow (see $\S 5$ ).

\section{Eddy structures}

\subsection{Dynamics}

We review here and in the following section the various ways in which progress in dynamical and statistical calculations are contributing to the basic problems of turbulence, set out in $\S 1$. Because the full flow field at high Re can neither be calculated analytically nor simulated numerically, various idealizations and approximations are made in constructing theoretical models. Note that even where complete simulations are possible (as they are for $R e \lesssim 10^{3}$ ), theoretical models are still being actively developed to understand the flow, to extend the statistical results to higher values of $R e$ and to provide methods for faster practical calculations.

There are two main theoretical approaches. One, described in this section, is to focus on the internal dynamics and external interactions of typical observed forms of eddy structure. In some studies the eddy flow fields are idealized in order to simplify the analysis. The other approach, described in $\S 5$, is to calculate in some simplified way the dynamics of an approximation of the overall flow field, usually in terms of its representation by a set of defined functions, e.g. its Fourier coefficients. The objective of either type of calculation is usually to derive or explain certain statistics of the whole flow, e.g. spectra, dissipation and transfer of energy and the probability distributions of the velocity field.

Studies of eddy structures are assisted by experiments and numerical simulations of the interactions between particular isolated vortical motions and surrounding flow fields that are characteristic of larger-scale structures within a turbulent flow (e.g. Couder*). The intrinsic assumptions involved in overall dynamic models (see §5), can be examined by studying how the turbulence responds to a narrow band of forcing 
frequencies or step perturbations (i.e. the relaxation process). Revealing experiments of this kind were conducted by Kellog \& Corrsin (1980) and and are now recommended as an essential element in improving models of turbulence spectra (Adrian $\&$ Moser 2000).

Much research continues to be based on the analysis of how small perturbations with random velocity and vorticity fields, $\boldsymbol{u}(\boldsymbol{x}, t), \boldsymbol{\omega}(\boldsymbol{x}, t)$, with an integral scale $l$ develop with time $(t)$ in a more energetic velocity and vorticity field $\boldsymbol{U}, \boldsymbol{\Omega}$ with length $L \gg l$ (Hunt*; Cambon*; Leonard*). Initially, the strain rate of the large-scale fields is greater than that of the small scale field $\boldsymbol{u}$, i.e. $(u / l) / S=\mu \ll 1$ where $S=U / L$ and $\mu$ is a small parameter. Studies along these lines show firstly how structures in the small-scale field evolve, secondly how they may react back on the large-scale field and thirdly how they affect the overall dynamics and statistics of turbulence (e.g. Cambon \& Scott 1999).

These stages can be explained in terms of the contributions of the large- and smallscale fields in the vorticity equation. The linear terms, caused by the direct interaction of the fields, whose magnitude can be characterized as $\gamma_{L} \mu S^{2}$, are $\nabla \wedge(\boldsymbol{U} \wedge \boldsymbol{\omega}+\boldsymbol{u} \wedge \boldsymbol{\Omega})$, and the nonlinear term caused by self-interaction of the small-scale fields, whose magnitude is $\gamma_{N L} \mu^{2} \boldsymbol{S}^{2}$ is $\nabla \wedge(\boldsymbol{u} \wedge \boldsymbol{\omega}-\langle\boldsymbol{u} \wedge \boldsymbol{\omega}\rangle)$, where the average operation is denoted by \langle\rangle as it must be taken over the large scale $L$. Here $\gamma_{L}$ and $\gamma_{N L}$ are coefficients of order unity that depend on the nature of the interaction.

The basic form of the large-scale straining may be characterized by its second-order invariant $I I$, where $I I$ is normalized on the squares of the symmetric strain ratio and the vorticity, i.e.

$$
I I=\frac{\left(\partial U_{i} / \partial x_{j}\right)\left(\partial U_{j} / \partial x_{i}\right)}{\Sigma^{2}+\frac{1}{2} \Omega^{2}}=\frac{\Sigma^{2}-\frac{1}{2} \Omega^{2}}{\Sigma^{2}+\frac{1}{2} \Omega^{2}},
$$

where

$$
\Sigma_{i j}=\frac{\partial U_{i}}{\partial x_{j}}+\frac{\partial U_{j}}{\partial x_{i}}, \quad \Sigma^{2}=\Sigma_{i j} \Sigma_{j i}, \quad \Omega^{2}=|\Omega|^{2} .
$$

It is also convenient to define the normalized third invariant

$$
I I I=\frac{\left(\partial U_{i} / \partial x_{j} \partial U_{j} / \partial x_{k} \partial U_{k} / \partial x_{i}\right)}{\left(\Sigma^{2}+\frac{1}{2} \Omega^{2}\right)^{2 / 3}} .
$$

Consider the case where $I I$ has a significant component, i.e. $I I+1>\mu$, then it is found that (except if $\partial U_{i} / \partial x_{j}$ is perfectly axisymmetric) the linear amplification of the non-uniform vorticity $\omega$ of the small scales leads to the formation of distinct thin layers, or sheets, parallel to $\omega$ and aligned in the direction of the strain, which rotate if $\boldsymbol{\Omega} \neq 0$ (e.g. Betchov 1956). This increases the magnitude of the nonlinear term and in general $\gamma_{N L}$ increases faster than $\gamma_{L}$. Since 'sheets' of small thickness tend to have a finite width, they begin to roll up and distort in other ways by self-induction (through the term $(\boldsymbol{u} \cdot \nabla) \omega)$ (Kida \& Tanaka 1994; Passot et al. 1995; Kevlahan \& Hunt 1997). Scalar fields are distorted by these motions into similar pattens of planar and rolled-up sheets (Brethouwer \& Nieuwstadt*).

The straining produced by the rolling-up weakens the part of the sheet that is feeding into the roll-up, which therefore tends to become an isolated vortex structure (e.g. Pullin \& Saffman 1998). In some circumstances if the initial small-scale velocity field is a coherent structure with a particular orientation and symmetry with respect to the axis of an irrotational strain, the nonlinear term does not grow as fast as the linear term (Gibbon*; see also Lundgren 1982) (i.e. $\gamma_{N L} \ll \gamma_{L}$ ), for example if wide vortex sheets or tubes are formed. In this special case the vorticity cannot grow without 
limit because the form of the small-scale flow is generally unstable to even smallerscale levels of fluctuation which can grow exponentially on this distorted but slowly changing structure, e.g. as Kelvin-Helmholtz-like billows, provided viscous effects are small enough locally (Passot et al. 1995). Both these types of nonlinearity, operating on the time scale $S^{-1}$, tend to limit the growth of $\overline{\omega^{2}}$ and to amplify those components of the velocity fluctuations which the linear distortion tends to suppress (e.g. normal to the mean velocity in a shear flow). The reduction of anisotropy by direct nonlinear mechanisms in straining flows leads to different results than statistical modelling based on the 'scrambling' process of Rotta (1951). For example the components amplified by the linear process are not correspondingly reduced by the nonlinear process.

The growth of $\overline{\omega^{2}}$ can also be limited by another mechanisms: when the stretching of the vorticity extends beyond the scale $L$ over which the large-scale strain is correlated, then the effective strain is weaker because it has a random orientation and magnitude (Leonard*). This limiting process also takes place on the strain time scale $S^{-1}$. The recent numerical study of Ohkitani (1998) shows that the mean-square growth of vorticity is less than that of the length of fluid elements $\overline{l^{2}}$, which is consistent with the existence of these self-limiting mechanisms.

The growth of the small-scale turbulence affects the non-uniformity of the largescale strain field (i.e. $\nabla \nabla \boldsymbol{U}$ ). This provides another mechanism for the limitation of the growth of small scales. The nonlinear self-induced terms $\nabla \wedge\langle\boldsymbol{u} \wedge \boldsymbol{w}\rangle$ at the large scale $L$ affect the vorticity on this scale, which can grow until a significant perturbation in the large structure $\boldsymbol{U}$ develops, such as a set of closed streamlines. This greatly limits the straining of the small scales (Kerr \& Dold 1994; Nazarenko, Kevlahan \& Dubrulle 1999). This example of an upscale process, which has been verified experimentally (Couder*), requires a stable large-scale flow with a significant amplitude for the largescale perturbation to develop driven by the small-scale turbulence. (See also Sulem et al. 1989.)

Where the large-scale straining is purely rotational, e.g. with vorticity $\Omega_{3}$, then $I I \simeq-1$. This motion has no direct stretching effect on the vorticity of the small-scale turbulence on a time scale $S^{-1}$; it merely rotates the vorticity and velocity fields which, for example, leads to oscillations in the ratios of the moments $\left(\overline{u_{1}^{2}} / \overline{u_{2}^{2}}\right)$ if the turbulence is initially anisotropic. On a longer time scale $T_{L}=L_{x} / u_{0}$, the small-scale turbulence increases the separation of fluid elements by a distance $\Delta_{3}$ in the direction of $\boldsymbol{\Omega}$. Since this nonlinear process amplifies the vorticity component $\omega_{3}$, in the direction of $\boldsymbol{\Omega}$, in regions where $\Delta_{3}>0$ and reduces $\omega_{3}$ in regions where $\Delta_{3}<0$, vortical structures emerge parallel to $\boldsymbol{\Omega}$. Furthermore, those having the same sense of vorticity as $\boldsymbol{\Omega}$ are stabilized against small fluctuations by the large-scale rotation, while those with the other sense do not tend to form and are unstable (Cambon*). Where these structures are formed very close to each other, they tend to rotate around each other and to merge into larger structures (e.g. Hopfinger, Browand \& Gagne 1982). This is one of several examples of where, as certain eddy structures form, they tend to merge with others nearby and suppress other types, both effects tending to amplify the local gradients of vorticity at the edges of the structures.

In simulations of the development of an initial distribution of vortices at $R e \simeq 0.150$, Ohkitani* showed how the vorticity was amplified in the form of sheets which then rolled up quite rapidly and, through viscous diffusion, turned into a distribution of elongated vortices or 'worms'. These persisted until the turbulence finally decayed. Despite the relative brevity of the sheet-roll-up phase, his calculations showed that this mechanism provided more of the transfer of energy to small scales than the longer lasting 'worm'-like phase. Perhaps this explains why the rolled-up vortex sheet 
in an extended straining flow (recently reviewed by Pullin \& Saffman 1998) can only persist and be continually regenerated at very high values of $R e$ (such as seen by traces in atmospheric and oceanic turbulence) and not at the lower values of $R e$ that can be directly simulated numerically.

Recent theory supported by numerical simulations and experiments has revealed more about the dynamics of these small but mature vortices, especially how they contribute locally to the role of dissipation of energy $\varepsilon$ and how, outside them, they induce helical streamlines and straining fields which affect other vortices (e.g. Okhitani*; Kida, Miura \& Adachi 2000; Douady et al. 1991, Vassilicos 2000a). It is found that these vortices have a finite length (which can be as large as the integral scale at low $R e$ ), are not very curved, and have a finite lifetime; these effects may be caused more by the growth of instabilities within the vortices stimulated by the random fluctuations in the surrounding flow than by strong mutual interactions between vortices (Verzicco \& Jiménez 1999; Melander \& Hussain (1993); Miyazaki $\&$ Hunt 2000). At high enough values of $R e$, this breakup stage could presumably be followed by a new cycle of sheet formation-roll-up-vortices-breakup; this could occur everywhere and at random places throughout the flow. The role of 'background' fluctuations outside the structure can be significant in all these stages (Hunt*; Tsinober 1998). Chertkov et al. (2000), and Ooi et al. (1999) have shown how different stages of this cycle can be mapped on to a graph of invariants of the velocity strain field (' $Q-R$ ' or $I I-I I I$ plots) from numerical simulations (see also Tsinober 1998). Hunt* suggested that this provides a semi-deterministic model for the natural time scale of the small-scale eddy motions.

Understanding how large or intense the structures grow and the different time scales at which they break-up affects how they contribute to the overall dynamics and statistics of the turbulence. Some investigators have suggested on theoretical grounds (Hunt*), others on the basis of studying vortices in two-dimensional flows (Kiya, Ohyama \& Hunt 1986) and low-order model behaviour of boundary layer eddies (Holmes et al. 1996), that most vortical structures move round each other, so that their interactions are long range and on average not very strong (see $\S 5$ ). On the other hand Hussain* and Moffatt* suggested that strong interactions (when the helicity integral of the structure changes) might occur sufficiently often to affect the dynamics and especially the extreme values in the probability distribution. A new analysis by Moffatt* of flow vortices grouped in two anti-parallel pairs and orientated so that they collide with each other at right angles showed how, even when viscosity is included in the calculation, the velocity and vorticity tend locally to an infinitely large value at a finite time $t_{\infty}$ that is independent of viscosity even when $R e$ is finite. This specific calculation of a realizable flow (assuming it remains stable and the critical symmetry of the vortices is exact) is consistent with some earlier theory that such singularities could exist (Leray 1933; Pumir \& Siggia 1990). Numerical simulations conducted during the programme by Ohkitani \& Gibbon (2000) showed that a class of stretched solutions identified by Gibbon, Fokas \& Doering (1999) leads to a finite-time singularity.

Mathematical studies are helping to define bounds and general properties of such singularities that form in a finite time. Doering \& Gibbon (2000) proved during their stay at the INI that there cannot be a finite-time singularity of the Navier-Stokes equation if the ratios between a set of statistically defined microscales, all smaller than the Taylor microscale, are increasing fast enough with time. Constantin (2000) obtained results on the inviscid Euler equations using the Cauchy-Weber relations (for the vorticity and velocity of distorted fluid line elements) which may point to the 
absence of finite-time, geometrically regular, self-similar singularities. The study of singularities and near-singularities is inherently linked to the pivotal dependence of dissipation on Reynolds number (Taylor's conjectured equation (1)) and to the way that this dependence is determined by the flow geometry of the underlying velocity field (see Doering \& Constantin 1998; Flohr \& Vassilicos 1997; Angilella \& Vassilicos 1999; Kerswell 1999).

The mechanisms for the growth and interaction of the largest-scale eddy structures are necessarily studied in the context of particular types of turbulent flows because these structures always retain some influence or 'memory' from initial or boundary conditions (see §5). Nevertheless, where the large-scale eddies are free to move and interact with each other, and whether produced homogeneously at some initial time or in some local region (as in a boundary layer), it is found that they have some general features in common. In both two- and three-dimensional turbulence there is a greater tendency for large vortical eddies to grow by the mechanism of boundary entrainment (discussed in $\S 3$ ) and by engulfing small eddies, than to be diminished by breakup caused by occasional collisions with other large eddies. There are stronger tendencies for three-dimensional as compared to two-dimensional vortices to become unstable and to interact (even at a distance) with other structures. The net growth rates of these structures is much less than for two-dimensional vortices, which is consistent with statistical results (e.g. Lesieur 1990). These mechanisms shed some light on the unresolved questions (Herring*; Davidson*) about the nature of longrange effects in two- and three-dimensional turbulence and the convergence of volume integrals $I_{k}=\int_{0}^{\infty} r^{k} R(r) \mathrm{d} r$ of the cross-correlations $R(r)=\overline{u(x) u(x+r)}$. Davidson* considered the angular momentum of large but finite volumes of isotropic turbulence, whose large eddies have small enough initial momentum that their energy spectrum $E_{(k)}$ is much less than $O\left(k^{2}\right)$ - Saffman (1967). He gave a new argument for why Loitsyanskii's (1939) integral $I_{4}$ is finite, and approximately constant. This integral plays a critical role in statistical models discussed in $\S 5$.

\subsection{Kinematics and statistics of eddy structures}

The objective identification of these 'structures' and the assessment of their contributions to the overall statistics of a flow is as important as their dynamics. There are three main approaches to the identification problem, according to the sampling method used and the type of eddy being analysed (Bonnet \& Glauser 1993). The first uses statistics, such as two-point Eulerian correlations to extract the forms of modes defined in fixed coordinate systems (e.g. Devenport's* study of eddies in the near wake). The second is based on measurements of individual realizations of the instantaneous fields. With only limited measurements available at high $R e$, well-focused identification methods using conditionally sampled data are necessary; new developments in the measurement and analysis of the multiple-scale properties and individual events using wavelet analysis were reported by Arneodo et al. (1999) and Nicolleau \& Vassilicos (1999) (see also Silverman \& Vassilicos 1999). When complete data of flow fields are available (which are only obtainable from numerical simulations and therefore at moderate values of $R e$ ), the forms of eddy structures can be evaluated in terms of tensorial invariants of the velocity gradient field ( $\$ 4.1)$. For example, vortices near the wall in boundary layers have been identified using as the threshold criteria various combinations of $I I, \Sigma^{2}, \Omega^{2}$ (Hussain*; Perry*; Lesieur*). Small-scale vortices in homogeneous turbulence were identified, for example as regions of low pressure, by Kida et al. (2000).

The third 'optimal' type of approach is to combine the first two, for example by 
using data at a point from a single realization, in combination with correlations from an ensemble, to infer the local eddy structure. To test the conjecture by Tabeling* that many vortices may exist close to each other, kinematic criteria would be needed. He linked this to Novikov's recent concept of a 'complex vortex' derived from conditionally averaged analysis of the Navier-Stokes equations.

Comparing different types of analysis, it appears that the 'modal' approach based on statistical data is most suitable for large eddies in inhomogeneous flows whose position in the flow is relatively fixed (e.g. close to the wall, or relative to the centre of a wake), and for analyzing their temporal evolution (Holmes et al. 1996). Other approaches are needed where the eddies move randomly and interact with others (Fung et al. 1992). In meteorology, where Lagrangian forecasts of the evolution and position of low-pressure vortical motion is one of the main objectives of modelling, variations in eddy structures have been identified in terms of changes in the capacity, or 'fractal' dimension (Methven \& Hoskins 1997) and to simple measures of the random movement of the structures in terms of their departure from straight-line trajectories over various periods of time (van der Waal \& Milton 1999).

Theoretical models, flow visualization experiments, wavelet analysis of measurements, and some numerical simulations, have all indicated how, for a scale less than the integral scale of turbulence $L_{x}$, eddies have various characteristic structures. Although there are differences between the mathematical representation of eddies, all methods tend to agree about their broad aspects such as the size and energy of the structure. However, at a detailed level, each method emphasizes different features; for example Fourier and wavelet transforms or spectra are sensitive to discontinuities and accumulation regions (Lundgren*; Leonard*), while multi-point cross-correlations describe the inhomogeneous structure and its dynamics (using proper orthogonal decompositions and modal dynamics). Hausdorff fractal dimensions, Hölder exponents (Eyinck*) and capacity dimensions (Vassilicos*) may be used to reveal scaling laws for 'wiggly' variables that are fractal and/or have accumulation regions with self-similar structures.

In some cases and for certain types of variables, the measures are related to each other. For example the exponent $\zeta$ in the cross correlation $\left\langle\Delta u^{2}(r)\right\rangle \sim r^{\zeta}$ is exactly related to the exponent $p$ in the spectrum $E(k) \sim k^{-p}$ (i.e. $p=\zeta+1$ ), and to the box counting dimension, $D_{k}^{\prime}$. In fact a smaller range of self-similar scales is required for one measure rather than the other (Vassilicos \& Hunt 1991). Other examples were discussed where the self-similarity can only be detected at finite $R e$ by plotting the data as one set of the higher moments against others (e.g. Ciliberto, Leveque \& Ruiz Chavaria 2000; Vulpiani et al. 2000; van der Water et al. 2000).

In some cases the exponent $p$ of the full spectrum $E(k)$ for all eddy scales is determined by the characteristic eddy spectrum, $E^{(e)}(k)$, i.e. $E(k) \propto E^{(e)}(k)$. This is consistent with measurements in turbulent shear flows (at moderate $R e$ ) where $p \simeq 2$ (Hunt \& Carruthers 1990) and the small scales in the inertial range of the turbulent flow (at very high $R e$ ) where $p \simeq 5 / 3$ (Moffatt 1984; Lundgren 1982). However, if the typical eddy structures for a range of scales are statistically independent (Perry*; Townsend 1976) and if the spatial and temporal distribution can be assumed or derived from studying their generation (cf. Belcher \& Vassilicos 1997) the spectrum may be largely determined by the distribution of eddies. In general this occurs when the distribution is quite flat and the singularities of the eddy spectrum are relatively weak. Perry* and Hunt* showed how 'space-filling' elongated wall vortices in a turbulent boundary layer imply that $E(k) \propto k^{-1}$, for a self-similar range of eddy scales larger than those in the inertial range. These questions are not settled; Hatakeyama 
\& Kambe (1992) have proposed a statistical model based on the independence of elemental line vortices, which is not consistent with studies indicating dynamical interactions of structures in the inertial subrange (e.g. Tsinober 1998).

It remains an open question whether, for the small scales, the full Lagrangian spectra are also determined by the eddy spectra, i.e. $\Phi^{L}(\omega) \simeq \Phi^{(L E)}(\omega) \propto \varepsilon \omega^{2}$ (Fung et al. 1992; Malik \& Vassilicos 1996). Such spectra are predicted by two-point closure models based on the Lagrangian renormalization approximation (Kaneda 1993; Kaneda*). Thomson* pointed out that the precise nature of these time-dependent processes at the eddy scale greatly affect $G_{\Delta}$, the normalized rate of separation of particles in the inertial range of turbulent flow, in particular the greater the degree of coherence the smaller the $G_{\Delta}$.

\section{Simulations and models of the full flow field}

We review here developments in the calculation of various approximations for individual realizations and statistics of the full flow field $\boldsymbol{u}$, especially for very high$R e$ flows. Approximations $\hat{\boldsymbol{u}}(\boldsymbol{x}, t)$ to the full flow field $\boldsymbol{u}(\boldsymbol{x}, t)$, with error $\delta \boldsymbol{u}(\boldsymbol{x}, t)$ are calculated either in order to simulate individual realizations for particular initial boundary conditions, as for example in environmental forecasting, or in order to derive from the realizations, whether analytically or by summation, statistics of the flow. This latter indirect approach is necessary when the statistics cannot be derived from 'statistical' models that derive these quantities directly. There is growing interest in different kinds of approximate models and simulations, including those based on governing equations other than those of Euler or Navier-Stokes. Furthermore, fast approximate simulation methods are of great practical use (e.g. Hanjalic*; Holm*; Rodi*). The basic dynamical statistical studies of eddy structure of the previous section can partly explain, and in some cases predict, the applicability of different approximate methods for various types of flow.

Some guiding principles, to help researchers and users alike, have been proposed based on the following set of non-dimensional parameters (Savill*; Hunt \& Savill 2002).

(i) When calculating a turbulent flow, like any other fluid flow, it is necessary to state the temporal and spatial domain. The dependence of the turbulence on the initial conditions, or upstream boundary conditions if the turbulence is advected into the domain, is defined by the residence time parameter $\alpha_{T}=T_{L} / T_{D}$, where the time a fluid particle has spent in the domain is $T_{D}$, and the natural time scale of energy-containing eddies is $T_{L}=L_{x} / u_{0}$. (Note that $\alpha_{T}$ may vary across the domain.)

(ii) The degree of non-equilibrium of the turbulence defined in a Lagrangian frame moving with mean velocity is $\alpha_{N E}=|P / \varepsilon-1| \simeq T_{L} / T_{D}$, where $P$ and $\varepsilon$ are the rates of production and dissipation of energy respectively. Here $T_{D}$ is the time scale of any imposed distortion of the turbulence, such as the time for elements to leave a turbulent boundary layer and enter the wake behind an obstacle, or be compressed in a shock wave - both being examples of aeronautical turbulent flows that are considered to be unsatisfactorily calculated by the present generation of practical models (Hills \& Gould*).

(iii) Turbulence dynamics are intrinsically inhomogeneous when the integral scale $L_{x}$ is of the order of or greater than the scale $\Lambda_{I}$ over which the turbulence structure varies (e.g. $\Lambda_{I}=\left(\overline{|\boldsymbol{u}|^{2}} / \nabla|\boldsymbol{u}|^{2}\right.$ or $\left.L_{x} / \nabla L_{x}\right)$ (Durbin*). The degree of this non-locality is defined by $\alpha_{N L}=L_{x} / \Lambda_{I}$. 
(iv) In large eddy simulations (LES) of the flow field it is usual to filter out the eddies with scales smaller than $l_{f}$, a measure of this approximation being $\alpha_{f}=\left(l_{f} / L_{x}\right)$.

(v) In some models of the full flow field and stochastic simulations small-scale dynamics are represented by random processes (Mason \& Thomson 1992) with a time scale $\tau_{s}$, a measure of this approximation being $\alpha_{s}=\tau_{s} / T_{L}$.

Note that these measures (i-v) are based on integral-scale quantities of turbulence because it is assumed that the models and simulations are being used primarily to calculate the mean flow and energy-containing eddies. To some extent these quantities also determine the smallest-scale motions even though the computations may not describe them exactly (e.g. on the Kolmogorov microscale $l_{k}$ and time scale $\tau_{k}$ ).

(vi) Most calculations are numerical and involve making approximations over spatial scales and temporal discretization scales $\Delta x, \Delta t$, a measure of the errors involved being $\Delta x^{*}=\Delta x / \Lambda_{I}$. Current research indicates how, although many earlier model calculations were greatly affected by numerical approximations, it is now possible to reduce $\Delta x^{*}$ and $\Delta t^{*}$ to sufficiently small values, thanks to greater computer power now available, and that most approximations or errors are caused by the model assumptions rather than their numerical approximations (Hills \& Gould*). Studies of the errors of the filtering and discretization approximations have shown that for largeeddy and stochastic simulations it is generally necessary that the discretization scales are smaller than the filtering and stochastic time scales (i.e. $\Delta x^{*} \lesssim \frac{1}{2} \alpha_{f} ; \Delta t^{*} \lesssim \frac{1}{2} \alpha_{s}$ ) (e.g. Mason \& Callen 1986; Guerts*).

There are three main areas of development in modelling the full flow field:

(a) answering some of the basic statistical questions about turbulence (such as those in $\S 1$ ) by reducing the number of assumptions made to calculate, at some defined level of accuracy, key statistical quantities (such as the $n$ th-moments of velocity at $p$ points, and $s$ time events $M^{(n ; p, s)}$;

(b) calculating low-order statistics (typically $n \leqslant 3 ; p \leqslant 2, s \leqslant 2$ ) for non-stationary, non-local turbulent flows near rigid boundaries and near interfaces with non-turbulent or other kinds of turbulent or fluctuating flows;

(c) approximate simulations, especially reducing the errors in the resolved scales, and improving the statistical or stochastic modelling of the subgrid-scale motions and its conditional dependence on the larger resolved scales.

Since turbulence has different physical and functional forms (e.g. in the relations between statistical properties) depending on the type of the flow (e.g. Lumley*), we now consider models in the context of these types, classified in terms of the parameters just introduced. General models and simulation methods need to be assessed over a range of turbulent flows, whereas those that are designed to be applicable in a narrow range should be assessed accordingly.

(a) Short time and rapidly changing turbulence $\left(\alpha_{T} \gg 1, \alpha_{N E} \sim 1, \alpha_{N L} \sim 1\right)$. For a short time $t>t_{0}$ after turbulence is generated within a domain or is advected into it (i.e. $\alpha_{T} \gg 1$ ) the nonlinear terms have only a small influence on the velocity field, while distortions by linear effects, such as by gradients of the mean velocity, impact on boundaries or body forces, may have a large effect. Note that, because of the linearity, the turbulence is mostly sensitive to the initial conditions, though for some types of distortion (such as mean shear) certain of the resulting turbulence statistics are quite insensitive to their initial state. The linearity enables many features of the turbulence to be calculated analytically, including even the first-order corrections in the nonlinear terms. Research into these rapidly changing flows is progressing in two main directions. First, increasingly complex forms of linear distortion are being analysed, particularly where the scale of the turbulence $L_{x}$ is larger than the distance 
$\Lambda_{T}$ over which mean strain is varying (Leonard*; Hunt \& Durbin 1999) (i.e. $\alpha_{N E} \gg 1$ ), or where combinations of distortion are being applied (e.g. mean shear and stable stratification) (Hanazaki*).

Second, several complex nonlinear effects can be studied because, when $\alpha_{T} \gg 1$, they are weak enough to be calculated by expansion methods, starting with the non-stationary inhomogeneous linear solutions. This enables the growth of the backreaction of the turbulence on the mean or larger scale flow to be estimated, including the growth of instabilities and waves, the development of large scale flows driven by Reynolds stresses of distorted turbulent flow fields, and the effects of the distortion on the nonlinear vortex stretching and advection (see $\S 4$ ). The effects of these terms may remain quite small for a large enough time (or distance) such that $\alpha_{T} \sim 1$, and the linear processes can effectively determine the flow structure even in fully developed flows. This is why these studies provide insights into fundamental mechanisms. They also have more practical objectives such as to provide an exact limiting case as a comparator for general statistical models or to calculate in detail the effects of distortion on particular flows with given upstream conditions.

(b) Statistical models for small-scale fully developed turbulence. There continues to be incremental progress in constructing 'theoretical-physics' models for calculating moments $M^{(n, p, m)}$ of the small-scale velocity field, from the Navier-Stokes equation, by making the fewest possible assumptions about the mechanisms and no explicit assumptions about numerical coefficients (McComb 1990). Although the ultimate aim is to describe the statistics completely (i.e. $n, p, m \rightarrow \infty$ ), at present the systematic renormalization and perturbation methods are limited to computing Eulerian moments with $n \leqslant 3, p \leqslant 2$, in particular the spectra in the Kolmogorov inertial and viscous ranges.

Earlier calculations in a fixed Eulerian frame using the direct interaction approximations (see Kraichnan 1959) needed to be modified to apply in a Lagrangian frame in order to agree with the observed form of the inertial-range spectrum, in which $E(k)=\alpha_{k} \bar{\varepsilon} k^{-p}$, where $p \simeq 5 / 3$. However in recent RNG calculations of McComb*, in which a different initial assumptions are made, this modification is not necessary. Although the amplitude $\alpha_{k}$ agrees well with the measurements to within the $10 \%$ accuracy of the experiments, there is no prediction about the small correction of $p$ dependent on the intermittency of the energy dissipation rate. Perhaps this is consistent with Kida's* conclusion that these methods provide satisfactory approximations for second- (and presumably other low-) order moments because they only depend on a subset of all the possible nonlinear interactions between Fourier modes or 'eddies'. These are not the most locally intense, such as those which lead to large fluctuations in the dissipation rate and the small corrections to $p$.

There remains a question as to whether or how these models, which assume independence between different components of the energy spectrum and in which the dynamical interactions are quite 'smooth', are consistent with models of the velocity fields of the local eddy structure (e.g. of rolled-up vortices) that may have the same form of energy spectra. One reason why they are not inconsistent is because the former perturbation model is only a valid solution to the governing equation for velocity fields averaged and therefore 'phase averaged' over time scales of the order of that of the local eddy scale $\tau(l) \sim \bar{\epsilon}^{-1 / 3} l^{+2 / 3}$, whereas local models fully satisfy these equations over local time scales that are much smaller. Therefore if the velocity field is analysed on a scale $\Delta x$, only eddy length scales of the order of $l$ would actually be represented, where $l \sim \Delta$. There are independent eddy motions smaller than $\Delta x$ and therefore these individual small eddies are smooth. However, as one observes in the 
different scales of motion in plumes and clouds, mathematically the filtered velocity is fractal in the sense that $\Delta u / \Delta x$ does not have a limiting value as $\Delta x / L_{x} \rightarrow 0$ and $\Delta x / \ell_{k} \rightarrow \infty$.

New approaches to statistical models for the higher moments $n \geqslant 3$ are being actively developed because of their intrinsic interest and because of their application in effectively defining the higher moments of the probability density function and the structure of internal intermittency. In other words they are more closely connected to the isolated extreme events, such as those discussed in $\S 3$. The analysis by Proccaccia* is a considerable extension of the Eulerian theory of third and higher two-point moments, i.e. $n=3, p=2$. If Kolmogorov's method of using the Kármán-Howarth equation (see Frisch 1995), which links second and third two-point moments, is reformulated to link higher moments, it is found that it is not mathematically consistent. This is because the higher moments are limited by viscous processes in the microscale range. However, new combinations of $n$ and $p$ can be defined that only depend on the inertial-range scales, and are therefore independent of the Reynolds number. No equivalent theoretical results have yet been derived for the cases of $n \geqslant 3$, $p \geqslant 2$. Proccaccia's approach is to extend the original approach of Richardson (1926) and Batchelor (1952) to develop, using extra assumptions, a Lagrangian analysis for the $n$th moment of the relative velocity of a fluid particle $\overline{\Delta u^{n}}(\Delta t)$, at a distance $\Delta t$ from the fractal trajectory of a reference particle.

Less ambitious and more local calculations on relative diffusion in a moving frame of reference have been developed by Pumir* (see $\S 3$ ) by considering the dynamics of small numbers of fluid particles moving together. Such calculations may lead to more information about the shapes of vorticity contours and relative velocities of particles, and better estimates for the fundamental constant $G_{\Delta}=\overline{\Delta^{2}}(t) / \varepsilon t^{3}$ (cf. $\S 4$ ).

There is still an active stream of turbulence research that is mainly based on the methods of statistical physics because of the difficulties of developing reductionist theories based on the equations of motion. One observational justification is that joint probability distributions for particular combinations of variables have similar forms in different types of turbulence (e.g. Chatwin*). The theoretical basis essentially follows from the arguments of Landau \& Lifschitz (1959) and Batchelor (1953), quoted in $\S 1$. As with other statistical research, the aim is to make general predictions based on a few physical or statistical assumptions. For example the conceptual Parisi-Frisch (1985) model for the formation of eddies as a fractal process shows consistency between recent measurements of higher-order moments $\overline{\Delta u^{n}}(r)$ for different values of $n(>3)$, and intermittency variables. Model differential equations for the probability distributions (and other stochastic variables?) are also providing links between these moments (Gawedski*). Each of such artificial statistical models has good and bad points; so far none of them can explain the differences between the moments of $\Delta u$ when the relative velocities are parallel to and perpendicular to the displacement vector $\boldsymbol{r}$ (van de Water*).

(c) Statistical models for the overall structure - two-point moments. Increasingly, the solutions of engineering and environmental problems involving turbulent flows require calculations of the spectra (or two-point moments) of the velocity. Also needed is some knowledge of the sensitivity of calculations of one-point moments (such as Reynolds stresses) to the variations in the spectra, anisotropy and inhomogeneity of the turbulence (a possible source of error emphasized in the account of one-point methods by Launder \& Spalding 1972). The most extensively developed model for spectra is the eddy damped quasi-normal Markovian (EDQNM) coupled differential equation model for the second- and third-order moment $M^{(2,2)}$. The basic theory 
(e.g. Lesieur 1990) includes some ideas from statistical physics, for example the relaxation time scale for relating second and third moments. For the case of isotropic turbulence, an alternative approach is to use renormalization methods, which avoids the assumption of this time scale (McComb 1990). EDQNM has been extended to uniformly distorted turbulent flows, such as large-scale compression, shear or stable stratification, etc. The method is consistent with linear theory for large strain rates and has been extensively verified (Cambon \& Scott 1999). In our notation the conditions for this locally homogeneous theory are $\alpha_{T} \sim 1 ; \alpha_{N E} \sim 1, \alpha_{N L} \ll 1$. Bertoglio* and Cambon* described how the method can be extended with the aid of further approximations to weakly inhomogeneous turbulence (i.e. $\alpha_{N L} \lesssim 1$ ) (when it is still possible to define three-dimensional spectra). It was shown how in some cases of strong deformation rate, such as during compression in an engine, one-point moments derived by integrating these spectra give more accurate calculations than those based on simpler equations for one-point models. This is because, except by adjustment of their empirical coefficients, such one-point models allow for similar variations in the turbulence structure (Launder \& Spalding 1972).

Where the turbulence is highly non-local or non-stationary, such as near rigid or flexible interfaces (i.e. $\alpha_{N L} \gg 1, \alpha_{N E} \gtrsim 1$ ) the above methods do not apply. However, linear methods can be used in some of these flows even though, formally, they are only valid when $\alpha_{T} \lesssim 1$. In some cases the nonlinear effects are suppressed and the turbulence is changing slowly with time. In other cases, a correction to the linear model can be estimated using a relaxation time that varies with the eddy scale (Mann 1994). Bannerjee* reviewed recent work showing how these methods largely predicted the turbulence structure near density interfaces, verified by experiments and direct numerical simulation.

(d) One-point closures for flows in engineering and the environment. In the majority of practical turbulent flow problems the main objective is still to calculate the mean velocity $\boldsymbol{U}(\boldsymbol{x}, t)$, temperature $\theta(\boldsymbol{x}, t)$, or some other mean scalar such as mass fraction, and some approximate measures of the amplitude and scale of the turbulence (e.g. $u_{0}=\sqrt{\overline{u_{1}^{2}}}$, and $L_{x}$ ). From the mean momentum equation it follows that the gradients of the Reynolds stresses $\overline{u_{i} u_{j}}$ should be calculated as accurately as the required gradients of $\boldsymbol{U}$, while other measures of turbulence may be derived less accurately (e.g. $\left.L_{x}\right)$. While $\overline{u_{i} u_{j}}(\boldsymbol{x}, t)$ are moments at one point, $L_{x}$ is formally a measure of twopoint moments. But it is often assumed to be a quantity defined at a point (assuming local homogeneity, i.e. $\alpha_{N L} \lesssim 1$ ) or estimated from the local value of mean dissipation $\varepsilon\left(\simeq u_{0}^{3} / L_{x}\right)$.

Practical models devised to predict these quantities have tended to be Eulerian and have not changed fundamentally over the past 25 years (Lumley*). Research into their rationale and limitations has led on to incremental modifications, usually designed for different types of turbulent flows. The numerous workshops for testing the models against experiments and systematic comparison of all the elements of the models with direct numerical simulations have all contributed to these advances. Following Prandtl's (1925) physical arguments based on his flow visualization studies of eddies in a shear flow, in the simplest statistical models the Reynolds stresses $\left(\overline{-u_{i} u_{j}}\right)$ are assumed to be proportional to the gradients of mean velocity $\partial U_{k} / \partial x_{j}$ and to the 'bremsweg' (later 'mischungsweg') length scale $l_{m}$. This depends on the type of flow and the location within it. Such relations, which are assumed to be independent of initial and boundary conditions of turbulence, and any non-local and non-stationary effects (i.e. $\alpha_{T} \ll 1, \alpha_{N L} \ll 1, \alpha_{N E} \ll 1$ ), are still widely applied for calculating nearly 
unidirectional flow because of their computational efficiency and convenience, for example over turbomachinery blades and in certain environmental flows. In many such cases the largest errors are caused by the 'wall-layer' approximations (e.g. equation (8)) in the boundary conditions for $U$ near smooth or rough rigid surfaces, which can only be derived by local analyses for the viscous processes in the former case or for the flow through the roughness elements in the latter case. In unidirectional shear flows the Reynolds stress $\overline{-u_{1} u_{3}}$ parallel to the mean shear $\partial U_{1} / \partial x_{3}$ is sometimes used to estimate the stresses in other directions and the total turbulence kinetic energy $K=\frac{1}{2} \overline{u_{i} u_{i}}$ on the assumption that they are proportional to the shear stress.

Where the mean flow and turbulence are changing rapidly (i.e. $\alpha_{N E} \sim 1$ ), the errors in these local equilibrium or 'mixing length' models are sometimes corrected by physically based modifications developed for the specific (e.g. meteorological) types of flow (Derbyshire et al.*). Usually for such flows more complex models are used which explicitly allow for finite values of $\alpha_{T}, \alpha_{N L}, \alpha_{N E}$. Following Kolmogorov (1942), and the later developments reviewed by Launder \& Spalding (1972) and Lumley (1978), time and space developments are calculated of either the turbulent kinetic energy $K=\frac{1}{2} \overline{u_{i} u_{i}}$ or the components of the Reynolds stress $\overline{u_{i} u_{j}}$. The factors considered are the gradients of the mean velocity, the fluctuating pressure gradients, the eddy-induced transport of turbulence across the shear flow, and the mean rate of dissipation $\bar{\varepsilon}$ by viscous processes. The last three terms have to be approximated in terms of the same second-order moments and the mean flow or by the use of additional equations. For general-purpose calculations of industrial and some environmental flows with a mean velocity that is large relative to the turbulent fluctuations (so that $u_{0} / U \ll 1$ ) but which may vary in direction, only two coupled differential equations for $K$ and $\bar{\varepsilon}$ are used, and often the Reynolds stresses are estimated by the local eddy viscosity approximation

$$
\left(\overline{u_{k} u_{k}} \frac{1}{3} \delta_{i j}-\overline{u_{i} u_{j}}\right)=C_{\mu}\left(K^{2} / \bar{\varepsilon}\right)\left(\partial U_{i} / \partial x_{j}+\partial U_{j} / \partial x_{i}\right),
$$

where $C_{\mu}$ is a coefficient determined by comparison with experiments.

For turbulent flows that are far from equilibrium, such as turbulence in a strongly diverging flow approaching a stagnation point (i.e. $\alpha_{N E} \gtrsim 1$ ), the turbulence structure changes rapidly, and the form of its anisotropy differs significantly from that in shear flows. Then the local relation (12) is incorrect and the $K-\varepsilon$ equations give quite misleading information about the turbulence. However, in using the full Reynolds stress transport equations (RSTE) (Hanjalic*; Craft*), $-\overline{u_{i} u_{j}}$ develops as a result of the history of the mean strain $\partial U_{i} / \partial x_{j}$. Some industries are making use of models based on these RSTEs (Laurence*), while others consider that the possibility of extra accuracy does not compensate for the extra complexity. In flows that are highly inhomogeneous (i.e. $\alpha_{N L} \gtrsim 1$ ) the higher relative contribution of the eddy transport, such as occurs in natural convection with low mean velocity (so that $u_{0} / U_{0} \lesssim 1$ ), can be estimated more accurately by calculating explicitly transport equations for the third moments that are otherwise approximated in the RSTE in terms of second moments (Andre et al. 1976; Ilyushin*; Launder*).

The $K-\varepsilon$ and RSTE model equations have been formulated quite generally so that all the tensors satisfy invariance properties and the dimensionless coefficients are the same in different types of flow and different ranges of $R e$. It is implicitly assumed that the effects of non-locality and non-equilibrium are small (i.e. $\alpha_{N L}<1, \alpha_{N E}<1$ ), and therefore that (cf. Launder \& Spalding 1972) any changes in the turbulence structure (e.g. spectra, anisotropy, etc.) only have a small effect on the second moments. However, in most recent developments, these assumptions about the universal and 
localized nature of second-order turbulence dynamics are being relaxed in various ways:

(i) The variations in the eddy structure of turbulent flow resulting from initial anisotropy of the moments $\overline{u_{i} u_{j}}$ (Townsend 1976), from their spectra or from various types of straining (cf. Cambon*), can be large enough to diminish the tendency of the pressure-strain term $\overline{p \partial u_{i} / \partial x_{j}}$ to reduce the anisotropy of the turbulence. This is now well understood in terms of the eddy dynamics (see $\S 3$ ), and can be succinctly described in an anisotropy diagram of the second and third variants $I I_{b}, I I I_{b}$ of the anisotropy tensor $b_{i j}=\left(\left(\overline{u_{i} u_{j}} / \overline{u_{k} u_{k}}\right)-\frac{1}{3} \delta_{i j}\right)$ (Lumley 1978) or of the anisotropy of the spectra (cf. Kassinos, Reynolds \& Rogers 2001).

This sensitivity is now allowed for in some models so that effectively the isotropic tendencies of the pressure-strain terms are assumed to be negligible if $I I I_{b}<0$ (Launder*).

(ii) Another way in which variations in the turbulence structure are being modelled within the same general methodology is (following Hanjalic, Launder \& Schiestel 1980; Schiestel 1987) to split the energy $(K)$ equation into two parts, one for calculating the large scales which are dependent on the production of energy by the mean shear and buoyancy and the transfer of energy to the small scales, and the other calculating the smaller scales. The differences in the development times of these two parts of the spectrum approximately model the effects of changes in the form of the energy spectrum on the large scale turbulence and the mean flow (Laurence*; Hanjalic*).

(iii) Where there are local gradients of the mean velocity $\partial U_{i} / \partial x_{j}$ the second 'fast' component of the pressure-strain terms is usually approximated as $K \boldsymbol{G}$ where $\boldsymbol{G}$ is a tensor proportional to the local value of $\left(\partial U_{i} / \partial x_{j}\right)$ (which naturally follows from the Poisson equation for the fluctuating pressure). However, where these mean gradients vary rapidly over the length scale of the turbulence (i.e. $\alpha_{N L} \gtrsim 1$ ), the assumptions of the modelling of these terms is invalidated. This causes errors, for example in the ratios of Reynolds stress $\overline{u_{1}^{2}} / \overline{u_{3}^{2}}$ etc. in high Reynolds number turbulence near a 'wall' and thence in calculations of heat transfer. One way of modelling this non-local effect is to allow quadratic and cubic products of Reynolds stress to appear in the linear rapid part of the pressure-strain term. This effectively accounts for the fact that mean strain distorts the shape of the two-point velocity correlation surfaces (which modify the pressure fluctuations). In this way one can formally arrange to satisfy the two-component limit to which turbulence reduces at a wall (Lumley 1978; Shih, Lumley \& Janicka 1987; Launder \& Li 1994; Craft, Ince \& Launder 1996; Craft*). To extend such calculations into the buffer layer, one approach is to introduce higher derivatives of the mean velocity gradients $\left(\partial^{2} U_{1} / \partial x_{3}^{2}\right.$ etc.) into the approximation for the pressure strain (Launder \& Li 1994; Craft*). Another approach is to introduce an auxiliary inhomogeneous differential equation for the variable $\boldsymbol{G}\left(x_{3}\right)$ with the right-hand side being proportional to $\partial U_{1} / \partial x_{3}$. This robust numerical approximation for the pressure Poisson equation is being applied in several engineering applications (Durbin*; Laurence*). It is consistent with the normal velocity eddies being blocked by the wall, which becomes an increasingly significant process at high $\operatorname{Re}$ (see $\S \S 2,3$ ). Similar non-local effects of blocking in stably stratified inversion layers (Banerjee*) have been handled by the two-component limit approach noted above (Craft et al. 1996; Launder*). It has alternatively been modelled in some atmosphere and ocean calculations by expressing the length scale (needed for momentum or scalar flux calculations such as (12)) as integral expressions which have to be evaluated implicitly (Bougeault \& Lacarèrre 1989). 
(iv) The relation (12) between Reynolds stress and mean velocity gradients, used in conjunction with the $K-\varepsilon$ pair of equations, is not only local, but also linear in these gradients. Calculations using linearized theory for rapidly changing turbulence (Townsend 1976) show that this approximation is also in doubt if the gradients change significantly on a time scale of the order of that of turbulence $T_{L}$ (i.e. $\alpha_{N E} \gtrsim 1$ ), which commonly occurs for example in aeronautical boundary layer flows. The errors are such that 'nonlinear eddy viscosity' formulations for (12) are being introduced in which $-\overline{u_{i} u_{j}}$ is expressed as an expansion, up to third order, in $\left\|\partial U_{i} / \partial x_{j}\right\|$ (Gatski*). However, even these steps are insufficient to represent correctly the sensitivity to streamline curvature. One proposal is that (12) be augmented by cubic-level products of mean strain and vorticity in ways that cannot simply be interpreted as modifying $C_{\mu}$ (Craft, Launder \& Suga 1997).

(e) Approximate simulations and derived statistics. There have also been interesting developments in the application of approximate calculations of individual realizations of turbulent flows. These methods are used mainly to derive statistics from their ensembles. Most of the research is focused on approximating the small scales, especially those close to boundaries, but because all simulations are limited by the size of the domain and the period of the computation, there continues to be some uncertainty about the approximations involved in simulating the largest scales of turbulence comparable to those of the space-time domain and in estimating the effects on these scales of the errors at the smaller scales.

For example, what is the effect on the statistical properties of boundary layer simulations if the domain is not large enough to model the eddy structures, which can be 18 times larger than the boundary layer thickness (see $\S 3$ ), which have not so far been observed in large-eddy simulations? However in some 'low-order' simulations such structures are postulated in the basic assumptions of the simulation (Holmes et al. 1996) and consequently are represented. If numerical simulations are used to calculate the space-time development of individual realizations of turbulent flow, then specific initial data are required. But they are incomplete in most applications such as environmental forecasting or for control of turbulent flows. Kreiss* posed the bold hypothesis that only the large-scale velocity field (with scale of order of $L_{x}$ ) need be specified (but at frequent time intervals much less than $T_{L}=L_{x} / u_{0}$ ) in order for the velocity field at small scales to adjust dynamically to the same form whatever its initial form, given the same large-scale field. He largely verified this conjecture in numerical simulations of two- and three-dimensional homogeneous turbulence forced at the large scale. The moderate Reynolds number, $R e \sim 200$, of the simulation may explain why no obvious small-scale instabilities were observed. This result is consistent with other studies, which show that large-scale eddy structures can dominate the evolution of the flow field and provide a rational basis for flow control (Holmes et al. 1996), at least in this range of $R e$. It may also be in accordance with the greatly reduced estimates for error growth in high-Re flow systems. When eddy structures are free to move, any 'error' in their position eventually grows algebraically, and not exponentially; this leads to lower growth in the errors of simulation of environmental flows than were originally estimated by Lorenz (1963). Indeed Kreiss went so far as to argue that a much smaller amount of data is required for environmental real time prediction than is usually being supplied! (But see Hunt 1999.)

Large-eddy simulations, which were reviewed by Sandham (1999), have for more than 30 years been producing striking new insights into turbulence, particularly the structure of the energy-containing eddies. Active research is still needed to calculate reliably the errors in the resolved motions on the scale of the filter $l_{F}$. Some new 
corrections have been proposed based on the assumption of similarity of eddy motion over a range of length scales (e.g. Geurts*). Although such filtering has some effect on the larger scales (so that the simulation cannot be a prediction for a particular realization far into the future), many tests show that because of the downscale cascade in three-dimensional turbulence, the structure and statistics of the large-scale eddy motion is insensitive to these small-scale modelling assumptions (Jiménez*). (The upscale cascade in two-dimensional turbulence makes the structures sensitive to smaller-scale processes, e.g. Dritschel 1993.) The interactions between large and small eddy motions are more critical near rigid boundaries within a surface layer of thickness $h_{s}$; shear layers and coherent eddy structures form which extend over distances parallel to the wall that greatly exceed $h_{s}$ and often exceed $L_{x}$. These affect the level of the turbulent fluctuations near the wall and the large-scale motions in the interior of the flows, especially in thermal convection. It is now realized that the earlier approach for modifying LES near a wall by damping the velocity components is quite incorrect at very high $\operatorname{Re}\left(\gtrsim 10^{4}\right)$ because the wall amplifies the small-scale turbulence. A replacement by a local, quasi-steady, boundary-layer velocity profile with associated small-scale turbulence (Thomson*; Moin*) at least provides a reasonable first-order correction. But this does not completely model the structure of the surface-layer eddies which is significant for calculating the transition between the turbulence in the boundary layer at the trailing edge of an aerofoil and in the wake. Currently, various attempts are being made to model the layer with local boundary layer equations so that the solutions match with the resolved motions above the surface (Moin*).

Because LES methods use grids that are much smaller than the integral scale (at least $1 / 10$ ) they require enormous computation time. Yet many studies show that for unsteady, non-local, turbulent flows, where $\alpha_{N L} \gtrsim 1$ and $\alpha_{N E} \gtrsim 1$, such as thermal convection with a low mean velocity or unsteady wake flows, the $K-\varepsilon$ or RSTE equations are too inaccurate to be used as statistical models (since they require $\left.\alpha_{N L} \ll 1, \alpha_{N E} \ll 1\right)$. However, these can be used as unsteady equations to calculate a very large-eddy simulation (VLES) approximation to the realization of the unsteady velocities of the large eddies. The 'turbulence' in the statistical equations is assumed to represent the small-scale turbulence in the VLES unsteady calculations. Thus the method involves an assumption about the independence of large- and smallscale motions. This assumption is similar to that made in LES about the resolved and 'subgrid' scale eddies. But in the case of VLES because more of the dynamics at the resolved scale are 'modelled' (e.g. eddy transport), the discretization scales and (implicit) separation scale are both much greater than for LES. This speed-up in computations more than compensates for any loss in accuracy for some flows (Hanjalic*), though others at the meeting disagreed.

As with other statistical models, appropriate approximations are made for specific flows to simplify the calculations sufficiently for particular features to be explored in more depth, for example by analytical solutions or using dynamical systems theory to study how the solutions evolve in time (Holmes et al. 1997). Research using these analytical solutions is showing how, below each large eddy, the surface layers of the interior flow have a complex internal structure at very high $R e$ that would need an unrealistically small grid size and large computational time to be completely resolved (cf. Plate et al. 1998).

The ways in which small scales affect calculations of the large-eddy structure are quite sensitive to the assumptions about the surface boundary conditions and the small-scale statistical models; for example it depends on whether mean streamwise vortical eddies or other secondary flows exist, driven by normal stresses of large-scale 
turbulence. It remains to relate these calculations (e.g. Townsend 1976) to LES and other direct simulations of the large-scale eddy structure.

All the previous approximate simulations were based on filtering, averaging and approximating the Navier-Stokes equation. However, by analogy with statistical models that resemble the stochastic phenomena of turbulence, there are also methods based on phenomenological arguments for simulating the random or mean velocity fields without solving or approximating the Navier-Stokes equations. These may provide the full field or partial elements of it, such as the random fluctuations smaller than the resolved scales of large-eddy simulations. (For a review of Monte Carlo or kinematic simulations see Elliott \& Majda (1996).) A novel approach for calculating the mean velocity in shear flows as $R e$ varies from $10^{3}$ to $10^{6}$ by heuristically adapting the Navier-Stokes equations to reflect the motion of large eddies conveying momentum across the shear flow has been proposed by Holm*. Its solution agrees to within $5 \%$ with measured profiles of the mean velocity over this range of $R e$.

\section{Concluding remarks}

Can we conclude that current research is making progress towards answering the main questions about turbulence? The evidence of this review suggests that the answer is yes, but that we are still some way off finding complete answers. Some particular advances are summarized in the abstract.

The main question on which hang many others, is to what degree fluid turbulence is a universal phenomenon. On the one hand, research continues to provide more evidence $(\S 3)$ and better dynamical explanations $(\S 4)$, though still no complete theory, for the occurrence of similar qualitative features at the smaller scales in all types of three-dimensional turbulent flows; namely random, intermittent motions on wide ranges of length and time scales, where the very smallest scales are determined in part by molecular processes, a net cascade of energy to these scales, and a mean rate of dissipation $\hat{\varepsilon}$, that, when normalized on large-scale motions, is approximately independent of the Reynolds number. On the other hand, there are many non-universal aspects; not only are there qualitative differences in the structure of the large-scale eddy motions, but there is also increasing evidence $(\S 3)$ that in quantitative terms (e.g. statistical two-point, Eulerian measures) the above small-scale phenomena depend to some degree on the particular types of large-scale flow. The dependence of the eddy's structure and statistics on the flow type is also found (both theoretically and experimentally) in the underlying dynamics that determines the eddy motions. This is reflected in the sensitivity to the type of flow of the space-time development of the moments, as for example in Reynolds stress transport equations (§5).

A consequence of this increasing evidence and understanding of non-universality is a change in the direction of research. There is now more emphasis on studying various types of turbulence within distinct parameter ranges, leading to a variety of statistical models and approximate simulations that typically depend on the characteristic forms of the large-eddy structures in each type.

At the same time, some models that have been formulated on the assumption of great generality are being adapted to particular types of flow, for example by allowing for significant non-local and non-equilibrium effects $(\S 5)$. Some industrial users of statistical models have called for their systematic classification in terms of their assumptions and ranges of validity, so as to provide initial guidance to those applying such models to any particular type of flow. It was suggested that research 
along these lines may be at least as useful as conducting further comparisons among very general models applied to test case flows, which inevitably are quite idealized in terms of the geometry of the flow and the types of initial and boundary conditions.

Nevertheless, the search for some kind of universality is the goal of much experimental research in high Reynolds number turbulence; it tends to be restricted to studies of the statistics of the relative velocity $\Delta u$ over very small length and time scales in the inertia range; evidence continues to support the generalized Kolmogorov hypothesis that the statistics of $\Delta u$ (when suitably normalized and corrected for large-scale motions) are approximately the same in all types of fully developed turbulence at very high $\operatorname{Re}\left(\gtrsim 10^{4}\right)$. Consequently much theoretical research is still directed towards establishing a dynamically based, universal statistical theory for motions in the inertial and viscous microscale ranges. Accurate Lagrangian and multipoint Eulerian measurements, which will require the new facilities now being proposed (e.g. Nieuwstadt*), could provide the critical tests for any such theory, especially if (see $\S 4)$ it is based on an analysis of the velocity field around moving fluid elements.

Our review shows that although, regrettably, there are rather few innovative experimental research projects, there is certainly a diversity of theoretical and computational methods in turbulence. Most of them contribute some insight into the varied manifestations of turbulence. It is noticeable that some authors explicitly estimate the range of validity of their methods and results, but many others still leave this task to the reader or, worse, the person applying the method in practice. Another reason for the variety of methods, and of the focus of research on statistical models and approximate simulations, is that $(\S 5)$ the scientific and practical applications vary greatly. Not only do the nonlinear interactions between turbulence and other processes vary, but also the level of complexity that is appropriate changes depending on the availability of computing capacity (for the turbulence part of the total calculation) and of sufficiently detailed or accurate input data.

Currently this diversity of methods and assumptions is not sufficiently understood or valued by those engaged in turbulence research and its applications. Some interesting combinations of different and hitherto competing approaches are now being tried, such as integrating the statistical and dynamical analyses of eddies by using Reynolds stress transport models in large-eddy simulations. At the same time, new hypotheses and general questions still have to be rigorously and competitively examined, such as those that emerged this year about the transition of very high Reynolds number eddy structure and the mathematical properties of singularities. Clearly this field of research is flourishing!

The Isaac Newton Institute Programme on Turbulence was generously supported by the following organizations: the Engineering and Physical Sciences Research Council (EPSRC), Royal Academy of Engineering, Rolls Royce, BAE SYSTEMS, DERA, Met Office, British Gas Technology and British Energy. Some workshops were sponsored by ERCOFTAC. Associated Companies were BNFL Magnox Generation, Schlumberger, Ove Arup, the major commercial code vendors, FLUENT Ltd, AEA Technology Ltd, CHAM Ltd. and Computational Dynamics Ltd. We are grateful for individual support as follows: J. C. R. H. to Trinity College, and Delft University of Technology; J.C.V. to the Royal Society. We are particularly grateful to Teresa Cronin without whom this paper would not have seen the light of day because she typed and retyped endless versions of it and carried out most of the reference search seemingly tirelessly. 


\section{REFERENCES}

Adrian, R. H. \& Moser, R. 2000 Report of a workshop on the development of large eddy simulation. www.uiuc.edu/People/Adrian.html.

André, J. C., De moor, G., Lacarrère, P. \& Du vachat, R. 1976 Turbulence approximations for inhomogeneous flows. J. Atmos. Sci. 33, 476-481.

Angilella, J. R. \& Vassilicos, J. C. 1999 Time-dependent geometry and energy distribution in a spiral vortex layer. Phys. Rev. E 59, 5427-5439.

Arneodo, A., Manneville, S., Muzy, J. F. \& Roux, S. G. 1999 Revealing a lognormal cascading process in turbulent velocity statistics with wavelet analysis. Phil. Trans. R. Soc. Lond. A 357, $2415-2438$.

Atkin, C.* (EPF, Southampton, DERA), Monkewitz, P. (Lausanne) \& SANDHAM, N. (Southampton) Breakdown to Turbulence and its Control - Introduction.

BAHT, G. S. \& Narasimha, R. 1996 A volumetrically heated jet: large-eddy structure and entrainment characteristics. J. Fluid Mech. 325, 303-330.

BANERJEe, S.* (U.C. Santa Barbara) Closure Strategies for Modelling Turbulent and Transitional Flows: Turbulence near density interfaces -2 .

Barenblatt, G. I. \& Chorin, A. J. 1998 New perspectives in turbulence: scaling laws, asymptotics, and intermittency. SIAM Rev. 40, 265-291.

BATChELOR, G. K. 1952 Diffusion in a field of homogeneous turbulence II. The relative motion of particles. Proc Camb. Phil. Soc. 48, 345-362.

BatcheloR, G. K. 1953 The Theory of Homogeneous Turbulence. Cambridge University Press.

BAYly, B. J. 1986 Three dimensional instabilities of elliptical flow. Phys. Rev. Lett. 57, 2160-2163.

Belcher, S. E. \& VAssilicos, J. C. 1997 Breaking waves and the equilibrium range of wind-wave spectra. J. Fluid Mech. 342, 377-401.

Bertoglio, J. P.* (Lyon) Models based on 2-point closures for inhomogeneous turbulence.

Betchov, R. 1956 An inequality concerning the production of vorticity in isotropic turbulence. J. Fluid Mech. 1, 497-504.

Bisset, D. K., Hunt, J. C. R., CAI, X. \& Rogers, M. M. 1998 Interfaces at the outer boundaries of turbulent motions. Annual Research Briefs, pp. 125-135, Center for Turbulence Research, Stanford University.

Bonnet, J.-P.* (Poitiers) Compressibility effects in free shear flows.

Bonnet, J. P. \& Glauser, M. N. (Ed.) 1993 Eddy Structure Identification in free Turbulent Shear Flows. Kluwer.

Borée, J., Marc, D., Bazile R. \& Lecordier, B. 1999 On the behaviour of a large scale tumbling vortex flow submitted to a compression. European Series in Applied and Industrial Mathematics; Vol. 7. http://www.emath.fr/Maths/Proc. ESAIM Proceedings.

Bougeault, P. \& LACARrère, P. 1989 Parameterisation of orography-induced turbulence in a meso-beta scale model. Mon. Wea. Rev. 117, 1872-1890.

Brasseur, J.* (Pennsylvania State) Physical-scale-space dynamics of intermittent Burgers shocklets.

Brethouwer, G. \& Nieuwstadt, F* (Delft) Mixing of weakly and strongly diffusive passive scalars in isotropic turbulence.

Brown, G. L. \& Thomas, A. S. W. 1977 Large structure in a turbulent boundary layer. Phys. Fluids Suppl. 20, S243.

Cambon, C.* (Ecole Centrale de Lyon) Turbulence Structure and Vortex Dynamics: Stability of vortex structures in a rotating frame.

Cambon, C. \& Scott, J. F. 1999 Linear and nonlinear models of anisotropic turbulence. Ann. Rev. Fluid Mech. 31, 1-53.

CARPENTER, P. W.* (Warwick) The use of wall compliance to control transition and turbulence.

Castaing, B., Gunaratne, G., Heslot, F., Kadanoff, L., Libchaber, A., Thomas, S., Wu, X., ZALESKI, S. \& ZANETTI, G. 1989 Scaling of hard thermal turbulence in Rayleigh-Bénard convection. J. Fluid Mech. 204, 1-30

Cazalbou, J. B., Spalart, P. R. \& Bradshaw, P. 1994 On the behaviour of two-equation models at the edge of a turbulent region. Phys. Fluids 6, 1797-1804.

Chatwin, P. C.* (Sheffield) Perspectives in the Understanding of Turbulent Systems: Can turbulence researchers learn from probability theory?

Chertkov, M., Pumir, A. \& Shraiman, B. I. 2000 Statistical geometry and Lagrangian dynamics 
in turbulence. In Intermittency in turbulent flows (ed. J. C. Vassilicos). Cambridge University Press.

Ciliberto, S.* (Lyon), Leveque, E. \& Ruiz Chavaria, G. 2000 Nonhomogeneous scalings in boundary layer turbulence. In Intermittency in turbulence (ed. J. C. Vassilicos). Cambridge University Press.

Constantin, P. 2000 Navier-Stokes equations and fluid turbulence. Proc. Intl Congr. of Industrial \& Applied Maths (ed. J. Ball \& J. C. R. Hunt). Clarendon.

Couder, Y.* (Paris) The interaction between stretching and rotation: from the laminar to the turbulent situations.

CRAFT, T. J.* (UMIST) Closure strategies for modelling turbulent and transitional flows; Closure modelling near two-component limit: recent developments in second moment closure for buoyancy affected flows.

Craft, T. J., Ince, N. \& Launder, B. E. 1996 Recent developments in second-moment closure for buoyancy-affected flows. Dyn. Atmos. Oceans 23, 99-114.

Craft, T. J. \& LaUnder, B. E. 1996 A Reynolds stress closure designed for complex geometries. Intl J. Heat Fluid Flow 17, 245-254.

Craft, T. J., Launder, B. E. \& Suga, K. 1997 The prediction of turbulent transitional phenomena with a non-linear eddy viscosity model. Intl J. Heat Fluid Flow 18, 15-28.

DAVEnPORT, A. G. 1961 The spectrum of horizontal gustiness near the ground in high winds. $Q . J$. Roy. Metl. Soc. LXXXVII, 194-211.

Davidson, P. A.* (Cambridge) Perspectives in the Understanding of Turbulent Systems: The role of angular momentum in isotropic turbulence and in 2-D turbulence.

Derbyshire, S., Thomson, D. J. \& Woods, N.* (Met Office, Bracknell, UK) Turbulent systems: Problems and opportunities: Atmospheric/environmental flows.

DeVEnPORT, W.* (VPI Virginia) Characteristic eddy decompositions in real and model turbulent wakes.

Doering, C. R. \& Constantin, P. 1998 Bounds for heat transport in a porous layer. J. Fluid Mech. 376, 263-296.

Doering, C. R. \& Gibbon, J. D. 2000 Scale separation and regularity of the Navier-Stokes equations. In Intermittency in Turbulent Flows (ed. J. C. Vassilicos). Cambridge University Press.

Douady, S., Couder, Y. \& Brachet, M. E. 1991 Direct observation of the intermittency of intense vorticity filaments in turbulence. Phys. Rev. Lett. 67, 983-986.

Dritschel, D. G. 1993 Vortex properties of two-dimensional turbulence. Phys. Fluids 5, 984-997.

Durbin, P.* (Stanford) Mathematics of closure bifurcation of equilibria in second-moment closure.

Elliott, F. \& MAJdA, A. J. 1996 Pair dispersion over an inertial range spanning many decades. Phys. Fluids 8, 1052-1060.

EYINK, G.* (Arizona) Intermittency in turbulent flows and other dynamical systems: intermittency in turbulence: what is it and does it matter?

Ferré, J. A., Mumford, J. C., Savill, A. M. \& Giralt, F. 1990 Three-dimensional large-eddy motions and fine-scale activity in a plane turbulent wake. J. Fluid Mech. 210, 371-414.

Ffowcs Williams, J. E., Rosenblat, S. \& Stuart, J. T. 1969 Transitions from laminar to turbulent flow. J. Fluid Mech. 39, 547-559.

Flohr, P. \& VAssilicos, J. C. 1997 Accelerated scalar dissipation in a vortex. J. Fluid Mech. 348, 295-317.

FrISCH, U. 1995 Turbulence; the Legacy of A. N. Kolmogorov. Cambridge University Press.

Frisch, U. (Ed.) 1998 Advances in Turbulence VII. Kluwer.

Fuehrer, P. L. \& Friehe, C. A. 1999 A physically-based turbulent velocity time series decomposition. Boundary-layer Met. 90, 241-295.

Fung, J. C. H., Hunt, J. C. R., Malik, N. A. \& Perkins, R. J. 1992 Kinematic simulation of homogeneous turbulent flows generated by unsteady random Fourier modes. J. Fluid Mech. 236, 281-318.

GARTSHORE, I. S. 1966 An experimental examination of the large-eddy equilibrium hypothesis. J. Fluid Mech. 24, 89-98.

GATSKI, T. B.* (NASA-Langley) Closure strategies for modelling turbulent and transitional flows: Linear/non-linear eddy viscosity models.

GawedzKI.* (Paris) Turbulent advection and breakdown of the Lagrangian flow. 
George, W. K.* (New York State) Perspectives in the understanding of turbulent systems: similarity and turbulent solutions to the Navier-Stokes equations; Perspectives in the understanding of turbulent systems: some new ideas for wall-bounded flows.

George, W. K. \& CASTiLlo, L. 1997 Zero-pressure-gradient turbulent boundary layer. App. Mech. Rev. 50 (12), 689-729.

GeURTS, B. J.* (Twente) Direct and large-eddy simulation: balancing errors in LES.

GeurTs, B. J. \& LeONARD, A. 1999 Is LES ready for complex flows? Rep. NI99009-TRB, Isaac Newton Institute, Cambridge, UK (to appear in Closure Strategies for Transitional and Turbulent Flows (ed. B. E. Launder \& N. D. Sandham), Cambridge University Press, 2001).

GibBon, J. D.* (Imperial College) Another look at Lundgren's transformation and stretched vortex solutions of 3D Navier-Stokes and Euler; Turbulence structure and vortex dynamics vorticity alignment in the three-dimensional Euler and Navier-Stokes equations.

Gibbon, J. D., Fokas, A. \& Doering, C. R. 1999 Dynamically stretched vortices as solutions of the Navier-Stokes equations. Physica D 132, 497.

Gledzer, Y. B., Dolzhansky, F. W., Obukhov, A. M. \& Pomonanev, V. M. 1975 An experimental and theoretical study of the stability of motion in an elliptical cylinder. zv. Atm, Oc. Phys. 11, 617-622.

Gyr, A., Kinzelbach, W. \& Tsinober, A. (Eds.) 1999 Fundamental Problematic Issues in Turbulence. Birkhauser.

Hanazaki, H.* (Tohoku) Turbulence structure and vortex dynamics: linear processes in unsteady stably stratified turbulence with mean shear.

HanjaLic, K.* (Delft) Closure strategies for modelling turbulent and transitional flows: modelling double diffusion phenomena; VLES approaches; Mathematics of closure expanding the frontiers of single-point RANS application: some recent experience at TU Delft (Transition, multi-scales and VLES).

Hanjalic, K., Launder, B. E. \& Schiestel, R. 1980 Multiple time-scale concepts in turbulent transport modelling. In Turbulent Shear Flows 2, pp. 36-49. Springer.

Hatakeyama, N. \& Kambe, T. 1992 Statistical laws of random strains and vortices in turbulence. Phys. Lett. Rev. 79, 1257-1260.

HeAley, J.* (Keele) Breakdown to turbulence and its control using asymptotic methods to make practical nonparallel stability calculations.

Herring, J.* (NCAR Boulder) Problems and issues in 2-D turbulence.

HewitT, G. F.* (London) Computational modelling of multidimentional phenomena in two-phase flow.

Hills, D* \& Gould, A.* (British Aerospace) The INI programme on turbulence: the British Aerospace view.

Hodson, H.* (Cambridge) Breakdown to turbulence and its control: late stage transition on the low pressure turbine

HögSTRÖM, U. 1990 Analysis of turbulence structure in the surface layer with a modified similarity formulation for near neutral conditions. J. Atmos. Sci. 47, 1949-1972.

Holm, D.* (Los Alamos) The Navier-Stokes alpha model for fluid turbulence: Subgrid scale modelling of fluctuation effects in the equations of motion.

Holmes, P., Lumley, J. L. \& Berkooz, G. 1996 Turbulence, Coherent Structures, Dynamical Systems and Symmetry. Cambridge University Press.

Hopfinger, E. J., Browand, F. K. \& Gagne, Y. 1982 Turbulence and waves in a rotating tank. J. Fluid Mech. 125, 505-534.

Hoxey, R. P. \& Richards, P. J. 1992 Spectral characteristics of the atmospheric boundary layer near the ground. First UK Wind Engineering Conference, Engineering Department, University of Cambridge, UK, September.

Huerre, P. \& Monkewitz, P. A. 1990 Local and global instabilities in spatially developing flows. Ann. Rev. Fluid Mech. 22, 473-537.

Hunt, J. C. R. 1995 Practical and fundamental developments in the computational modelling of fluid flows. T. Hawksley memorial lecture at the Inst. Mech. Eng., 13th December 1994. J. Mech. Engng Sci. C 209, 297-314.

Hunt, J. C. R. 1999 Environmental forecasting and turbulence prediction. Physica D 133, 270-295.

HunT, J. C. R.* (UCL) Vortex dynamics and statistics of eddies in turbulence. 
Hunt, J. C. R. \& Carruthers, D. J. 1990 Rapid distortion theory and the problems of turbulence. J. Fluid Mech. 212, 497-532.

Hunt, J. C. R. \& Durbin, P. 1999 Perturbed vortical layers and shear sheltering. Fluid Dyn Res. 24, 375-404.

Hunt, J. C. R., Kaimal, J. C. \& GaYnor, J. E. 1988 Eddy structure in the convective boundary layer - new measurements and new concepts. Q. J. R. Met. Soc. 114, 821-858.

Hunt, J. C. R., Moin, P., Lee, M, Moser, R. D., Spalart, P., Mansour, N. N., Kaimal, J. C. \& GAYNOR, E. 1989 Cross correlation and length scales in turbulent flows near surfaces. Advances in Turbulence 2 (ed. H. Feidler \& H. Fernholtz), pp. 128-134. Springer.

Hunt, J. C. R. \& Morrison, J. F. 2000 Eddy structure in turbulent boundary layers. Eur. J. Mech. B/Fluids 19, 673-694.

Hunt, J. C. R. \& Savill, A. M. 2002 A guide to engineering turbulence models. In preparation.

Hunt, J. C. R. \& Vassilicos, J. C. 2000 Turbulence Structure and Vortex Dynamics. Cambridge University Press.

Hussain, F.* (Houston) Turbulence structure and vortex dynamics: core dynamics instability of a vortex in shear: a physical-space cascade mechanism; Breakdown to turbulence and its control: genesis and dynamics of coherent structures in near-wall turbulence and their control for drag reduction.

INOUE, E. 1951 On turbulent diffusion in the atmosphere. J. Met. Soc. Japan 29, 246.

ILYUSHIN, B.* (Russian Academy of Science) Closure strategies for modelling turbulent and transitional flows; use of higher moments to construct pdf's in stratified flows.

JiMÉNEZ, J.* (Madrid) Limits of performance of eddy viscosity subgrid models.

Jiménez, J., Wray, A. A., Saffman, P. G. \& Rogallo, R. S. 1993 The structure of intense vorticity in isotropic turbulence. J. Fluid Mech. 255, 65-90.

KanedA, Y. 1993 Langrangian and Eulerian time correlations in tubulence. Phys. Fluids A 5, $2835-2845$.

KANEDA, Y.* (Nagoya) Spectral closure approach - (non)-universality of the equilibrium range of turbulence in $2 \mathrm{D} / 3 \mathrm{D}$

KÁRmÁn, TH. vON 1930 Mechanische Ähnlichkeit und Turbulenz. Nachr. Ges. Wiss. Göttingeh, Math. Phys. K1, 58-76.

Kassinos, S. C., Reynolds, W. C. \& Rogers, M. M. 2001 One-point turbulence structure tensors. $J$ Fluid Mech. 428, 213-248.

Kellog, R. M. \& Corrsin, S. 1980 Evolution of a spectrally local disturbance in grid-generated, nearly isotropic turbulence. J Fluid Mech. 96, 641-669.

Kerr, R. M.* (NCAR) Turbulence structure and vortex dynamics: Applications of vortex dynamics to MHD current dynamics; Direct and large eddy simulation: dynamic backscatter tests of decaying isotropic turbulence.

Kerr, O. S. \& Dold, J. W. 1994 Periodic steady vortices in a stagnation-point flow. J. Fluid Mech. 276, 307-325.

Kerswell, R. R. 1999 A variational principle for the Navier-Stokes equations. Phys. Rev. E 59, $5482-5494$.

Kevlahan, N. K. R. \& Hunt, J. C. R. 1997 Nonlinear interactions in turbulence with strong irrotational straining. J. Fluid Mech. 337, 333-364.

Kholmyansky, M. \& Tsinober, A.* (Tel Aviv) 2000 On the origins of intermittency in real turbulent flows.

KIDA, S. 1998 Turbulence (in Japanese).

KidA, S., Miura, H. \& AdACHI, T. 2000 Flow structure visualization by low-pressure vortex. In Intermittency in Turbulent Flows (ed. J. C. Vassilicos). Cambridge University Press.

Kida, S. \& TANaKa, M. 1994 Dynamics of vortical structures in a homogeneous shear flow. J. Fluid Mech. 274, 43-68.

Kim, K. C. \& Adrian, R. J. 1999 Very large-scale motion in the outer layer. Phys. Fluids 11, 417-422.

Kit, E. L. G., Strang, E. J. \& Fernando, H. J. S. 1997 Measurement of turbulence near shear-free density interfaces. J. Fluid Mech. 334, 219-234.

Kiya, M., Ohyama, M. \& Hunt, J. C. R. 1986 Vortex pairs and rings interacting with shear layer vortices. J. Fluid Mech. 172, 1-15. 
Kolmogorov, A. N. 1941 Local structure of turbulence in an incompressible fluid at very high Reynolds numbers. Dokl. Akad. Nauk SSSR 30, 299-303.

Kolmogorov, A. N. 1942 Equations of turbulent motion of an incompressible fluid (Short resumé of report delivered at Kazan during the period 26-28 January 1942. Translation from the Russian by D. B. Spalding). Izv Akad. Nauk SSSR, Seria Fizicheska VI 1-2, 56-58.

Kolmogorov, A. N. 1962 A refinement of previous hypotheses concerning the local structure of turbulence in a viscous incompressible fluid at high Reynolds number. $J$ Fluid Mech. 13, 82-85.

KreIss, H.-O.* (UCLA) A numerical investigation of the interaction between the large and small scales of the two-dimensional incompressible Navier-Stokes equations.

Kraichnan, R. H. 1959 The structure of isotropic turbulence at very high Reynolds numbers. J. Fluid Mech. 5, 497-543.

LANDaU, L. E. \& Lifschitz, L. M. 1959 Fluid Mechanics. Pergamon.

LANGFord, J. \& Moser, R. D. 1999 Optimal LES formulations for isotraopic turbulence. J. Fluid Mech. 398, 321-346.

LAUNDER, B. E.* (UMIST) Application of TCL modelling to stably stratified flows.

LAUNDER, B. E. \& LI, S. P. 1994 On the elimination of wall topography parameters from secondmoment closure. Phys Fluids, 6, 999-1006.

Launder, B. E. \& Sandham, N. D. 2001 Closure Strategies for Transitional and Turbulent Flows. Cambridge University Press.

Launder, B. E. \& Spalding, B. 1972 Mathematical Models of Turbulence. Academic.

LAURENCE, D.* (Laboratoire National d'Hydraulique) Closure strategies for modelling turbulent and transitional flows: Closure modelling for industrial flows; LES modelling of industrial flows.

Le Dizes, S.* (Marseille) Perspectives in the understanding of turbulent systems: stability of vortex filaments in strain fields.

LEONARD, A.* (CALTECH) Turbulence structure and vortex dynamics: Evolution of localized packets of vorticity in turbulence; Direct and large-eddy simulation: a tensor-diffusivity subgrid model for large-eddy simulation.

LeraY, J. 1933 Etude de diverses équations intégrales non-linéaires et de quelques problèmes que pose l'hydrodynamique. J. Math. Pures Appl. 12, 1-82.

Lesieur, M. 1990 Turbulence in Fluids, 2nd Edn. Kluwer.

Lesieur, M. 1999 Turbulence et Determinisme. Presse Université de Grenoble.

LESZCHINER, M** (UMIST) Turbulence modelling.

LeweKe, T. \& Williamson, C. H. K. 1998 Cooperative elliptic instability of a vortex pair. J. Fluid Mech. 360, 85-119.

Lifschitz, A. \& Hameiri, E. 1991 Local stability conditions in fluid dynamics. Phys. Fluids A 3, 2644-2651.

LiN, C. C. \& ReID, W. H. 1959 Turbulent flow, theoretical aspects. In Encyclopaedia of Physics: Fluid Dynamics II (ed. S. Flugge \& C. Truesdell), pp. 438-523. Springer.

Lingwood, R.* (Cambridge) Breakdown to Turbulence and its control: Swept-wing boundary layers: convectively or absolutely unstable?

LoitsyAnski, L. G. 1939 Some basic laws for isotropic turbulent flow. Trudy Tsentr. Aero.-Giedrodin. Inst. 440, 3-23.

Lorenz, E. N. 1963 Deterministic nonperiodic flow. J. Atmos. Sci. 20, 130-141.

LuCCHINI, P.* (Milan) Transition prediction methods. Receptivity to low-frequency disturbances.

Lumley, J. L. 1978 Computational modeling of turbulent flows. Adv. Appl. Mech. Rev. 18, 124-176.

LumLeY, J. L.* (Cornell) Prediction methods in turbulence.

LundGREN, T. S. 1982 Strained spiral vortex model for turbulent fine structure. Phys. Fluids 25, $2193-2203$.

Lundgren, T. S.* (Minnesota) Perspectives in the understanding of turbulent systems: review of the spiral vortex turbulence model.

Lundgren, T. S. \& Mansour, N. N. 1997 Transition to turbulence in an elliptical vortex. J. Fluid Mech. 307, 43-62.

Malik, N. A. \& VassiLicos, J. C. 1996 Eulerian and Lagrangian scaling properties of randomly advected vortex tubes. J. Fluid Mech. 326, 417-436.

Malkus, W. V. R. \& WALEFFE, F. 1991 Transition from order to disorder in elliptical flow: a direct 
patch to shear flow turbulence. In Advances in Turbulence 3 (ed. A. V. Johansson \& P. H. Alfredsson). Springer.

ManN, J. 1994 The spatial structure of neutral atmospheric surface-layer turbulence. J. Fluid Mech. 273, 141-168.

Maruší́, I. \& Perry, A. E. 1995 A wall-wake model for the turbulence structure of boundary layers. Part 2. Further experimental support. J. Fluid Mech. 298, 389-407.

Mason, P. J. \& Callen, N. S. 1986 On the magnitude of the subgrid-scale eddy coefficient in large-eddy simulations of turbulent channel flow. J. Fluid Mech. 162, 439-462.

Mason, P. J. \& Thomson, D. J. 1992 Stochastic backscatter in large-eddy simulations of boundary layers. J. Fluid Mech. 242, 51-78.

МсСомв, W. D. 1990 Theory of Fluid Turbulence. Clarendon.

МсСомв, W. D.* (Edinburgh) Can microscopic RG theory be applied to the large-eddy simulation of macroscopic fluid turbulence?; Testing the 'hypothesis of local chaos' by calculating a renormalized dissipation rate for isotropic turbulence; Renormalized perturbation theory: has the turbulence problem been solved?

McGrath, J. L., Fernando, H. J. S. \& Hunt, J. C. R. 1997 Turbulence, waves and mixing at shear-free density interfaces. Part 2. Laboratory experiments. J. Fluid Mech. 347, 235-261.

Melander, M. V. \& Hussain, F. 1993 Coupling between a coherent structure and fine-scale turbulence. Phys. Rev. E. 48, 2669-2689.

Methven, J. \& Hoskins, B. J. 1997 Spirals in potential vorticity. Part I: measures of structure. J. Atmos. Sci. 55, 2053-2066.

MiyazaKi, T. \& Hunt, J. C. R. 2000 Turbulence structure around a columnar vortex; rapid distortion theory and vortex wave excitation. J. Fluid Mech. 402 349-378.

Moffatt, H. K.* (Cambridge) The Burgers vortex: variations on a turbulent theme.

MofFATT, H. K. 1984 Simple topological aspects of turbulent vorticity dynamics. In Turbulence and Chaotic Phenomena in Fluids (ed. T. Tatsumi). Elsevier.

Morn, P.* (Stanford) Some future challenges in large eddy simulation.

Moin, P. \& Mahesh, K. 1999 Direct numerical simulation: A tool in turbulence research. Ann. Rev. Fluid Mech. 30, 539-578.

Monin, A. S. \& Yaglom, A. M. 1975 Statistical Fluid Mechanics. MIT Press.

Moser, R. D., Langford, J. A. \& Volker, S. 1999 Optimal LES: How good can an LES be? Proc. Second AFOSR Intl. Conf. on DNS and LES, June 7-9, Rutgers University.

Moser, R. D., Rogers, M. M. \& EwING, D. W. 1998 Self-similarity of time-evolving plane wakes. J. Fluid Mech. 367, 255-289.

Mydlarski, L. \& Warhaft, Z. 1998 Passive scalar statistics in high Péclet-number grid turbulence. J. Fluid Mech. 358, 135-175.

Nazarenko, S., Kevlahan, N. K.-R. \& Dubrulle, B. 1999 WKB theory for rapid distortion of inhomogeneous turbulence. J. Fluid Mech. 390, 325-348.

Nicolleau, F. \& Vassilicos, J. C. 1999 Wavelets for the study of intermittency and its topology. Phil. Trans. R. Soc. Lond. A 357, 2439-2457.

Nie, Q. \& TANVEer, S. 1999 A note on third-order structure functions in turbulence. Proc. R. Soc. Lond. A 455, 1615-1635.

NieuwstadT, F.* (Delft) Future strategies towards understanding and prediction of turbulent systems: A 200 bar wind tunnel for turbulence measurements at very high Reynolds numbers.

Noullez, A., Wallace, G., Lempert, W., Miles, R. B. \& Frisch, U. 1997 Transverse velocity increments in turbulent flow using RELEIF technique. J. Fluid Mech. 339, 287-307.

OhKitani, K. 1998 Stretching of vorticity and passive vectors inisotropic turbulence. J. Phys. Soc. Japan 67, 44-47.

OhKitani, K.* (Kiyoto) Perspectives in the understanding of turbulent systems: Characterization of nonlocality in turbulence. Informal discussions on triad interactions in turbulence; Triad interactions in forced turbulence.

Ohkitani, K. \& Gibbon, J. D. 2000 Numerical study of singularity formation in a class of Euler and Navier-Stokes flows. Phys. Fluids 12, 3181-3194.

Ooi, A., Martin, J., Soria, J. \& ChOng, M. S. 1999 A study of the evolution and characteristics of the invariants of the velocity-gradient tensor in isotropic turbulence. J. Fluid Mech. 381, $141-174$

PARISI, G. \& FrISCH, U. 1985 On the singularity structure of fully developed turbulence. In 
Turbulence and Predictability in Geophysical Fluid Dynamics, Proc. Intl. School of Physics. 'E. Fermi', 1983, Varenna, Italy (ed. M. Ghil, R. Benzi \& G. Parisi), pp. 84-87. North-Holland.

Passot, T., Politano, H., Sulem, P. L., Angillela, J. R. \& Meneguzzi, M. 1995 Instability of strained vortex layers and vortex tube formation in homogeneous turbulence. J. Fluid Mech. 282, 313-338.

Perry, A. E.* (Melbourne) Breakdown to turbulence and its control: turbulent spots; Mathematics of closure: closure for the streamwise evolution of turbulent boundary layers using classical similarity laws and structure based modelling.

Perot, B. \& Moin, P. 1995a Shear-free turbulent boundary layers. Part 1. Physical insights into near wall turbulence. J. Fluid Mech. 295, 199-227.

Perot, B. \& Moin, P. $1995 b$ Shear-free turbulent boundary layers. Part 2. New concepts for Reynolds stress modelling of inhomogeneous flows. J. Fluid Mech. 295, 229-245.

Plate, E. J., Federovich, E. E., Viega, D. X. \& Wyngaard, J. C. (Eds.) 1998 Buoyant Convection in Geophysical flows. Kluwer.

Prandtl, L. 1925 Bericht über Untersuchung zur Ausgebildeten Turbulenz. Z. Angew. Math. Mech. 5, 136-139.

Praskovsky, A. A., Gledzer, E. B., Karyakin, M. Yu. \& Zhou, Y. 1993 The sweeping decorrelation hypothesis and energy-inertial scale interaction in high Reynolds number flows. J. Fluid Mech. 248, 493-511.

Procaccia, I.* (Weizmann Inst) Perspectives in the understanding of turbulent systems: The calculation of the anomalous scaling exponents in turbulence from first principles.

Pullin, D. I. \& Saffman, P. G. 1998 Vortex dynamics in turbulence. Ann. Rev. Fluid Mech. 30, $31-51$.

Pumir, A.* (INLN, Nice) Geometry of Lagrangian dispersion in turbulence.

Pumir, A. \& Siggia, E. D. 1990 Collapsing solutions in the 3D Euler equations. Phys. Fluids A 2, $220-241$.

Queiros-Conde, D. \& VAssilicos, J. C. 2000 Turbulent wakes of 3-D fractal grids. In Intermittency in Turbulent Flows (ed. J. C. Vassilicos). Cambridge University Press.

REEKS, M.* (ISIS) Closure approximations for dispersed particle flows.

Richardson, L. F. 1926 Atmospheric diffusion shown on a distance-neighbour graph. Proc. R. Soc. Lond. A 110, 709-737.

RoDI, W.* (Karlsruhe) Closure strategies for modelling turbulent and transitional flows: large-eddy simulation of bluff body flows.

RotTA, J. 1951 Statistische theorie nichthomogeneous turbulenz. Z. Phys. 129, 547.

Ruelle, D. \& Takens, F. 1971 On the nature of turbulence. Commun. Math. Phys. 20, 167-192.

Saddoughi, S. G. \& Veeravalli, S. V. 1994 Local isotropy in turbulent boundary layers at high Reynolds number. J. Fluid Mech. 268, 333-372.

SAfFman, P. G. 1967 The large scale structure of homogeneous turbulence. J. Fluid Mech. 27, 581-589.

SANDHAM, N. D. 1999 A review of progress on direct and large-eddy simulation of turbulence. Isaac Newton Institute Rep. NI99013-TRB.

SAndham, N. D.* (Southampton) Perspectives in the understanding of turbulent systems: Exploiting direct numerical simulation of turbulence. Closure strategies for modelling turbulent and transitional flows: DNS studies of compressible flows.

Sandham, N. D. \& Kleiser, L. 1992 The late stages of transition to turbulence in channel flow. J. Fluid Mech. 245, 319-348.

SAvill, A. M.* (Cambridge) Breakdown to turbulence and its control: Introduction to the COST/ERCOFTAC modelling workshop. Closure strategies for modelling turbulent and transitional flows: By-pass transition using conventional closures transition -2 ; New strategies in modelling by-pass transition.

SCHIESTEL, R. 1987 Multiple time-scale modeling of turbulent flows in one-point closures. Phys. Fluids 30, 722-731.

SchWARZ, K. W. 1990 Evidence for organised small scale structure in fully developed turbulence. Phys. Rev. Lett. 64, 415-418.

ShiH, T.-H., Lumley, J. L. \& JANICKA, J. 1987 Second-order modelling of a variable-density mixing layer. J. Fluid Mech. 180, 93-116.

Silverman, B. W. \& VAssilicos, J. C. (Eds.) 1999 Proceedings of the Royal Society Discussion 
Meeting on Wavelets: the key to intermittent information? Phil. Trans. R. Soc. Lond. A 357, $2393-2625$.

Smith, F.* (UCL) On nonlinear spots in favourable or adverse pressure gradients.

Sreenivasan, K. R.* (Yale) On structures and statistics in small scale turbulence; Polymers' effects on turbulence.

Sreenivasan, K. R. \& Antonia, R. A. 1998 The phenomenology of small scale turbulence. Ann. Rev. Fluid Mech. 29, 435-472.

Sulem, P. L., She, Z. S., Scholl, H. \& Frisch, U. 1989 Generation of large-scale structures in three-dimensional flow lacking parity-invariance. J. Fluid Mech. 205, 341-358.

TABeling, P.* (ENS Paris) Perspectives in the understanding of turbulent systems: Cascades in 2-D turbulence; New scales in three dimensional turbulence.

TaYlor, G. I. 1938 Production and dissipation of vorticity in a turbulent fluid. Proc. Roy. Soc. Lond. A 164, 15-32.

TenNekes, H. 1975 Eulerian and Lagrangian time microscales in isotropic turbulence. J. Fluid Mech. 67, 561-567.

TenneKes, H. \& Lumley, J. L. 1971 First Course in Turbulence. MIT Press.

Thomson. D. J.* (Meteorological Office) Understanding and predicting many environmental flows requires consideration of turbulence.

Townsend, A. A. 1976 Structure of Turbulent Shear flow. Cambridge University Press.

Trefethen, L. N., Trefethen, A. E., Reddy, S. C. \& Driscoll, T. A. 1993 Hydrodynamic stability without eigenvalues. Science 261, 578-584.

Tsinober, A.* (Tel Aviv) How relevant are Burgers-like objects to/for turbulence.

TsinOBER, A. 1998 Is concentrated vorticity that important? Eur. J. Mech. B 17, 421-449.

Tsinober, A. (Tel Aviv) 2000 Vortex stretching versus production of strain/dissipation. In Vortex Dynamics and Turbulence Structure (ed. J. C. R. Hunt \& J. C. Vassilicos), Cambridge University Press.

TURNER, J. S. 1986 Turbulent entrainment: the development of the entrainment assumption, and its application to geophysical flows. J. Fluid Mech. 173, 431-471.

VAN ATTA, C. 1991 Local isotropy of the smallest scales of turbulent scaler and velocity fields. Proc. R. Soc. Lond. A 434, 139-147.

VAssilicos, J. C. $2000 a$ Near-singular flow structure: dissipation and deduction. In Vortex Dynamics and Turbulence Structure (ed. J. C. R. Hunt \& J. C. Vassilicos). Cambridge University Press.

Vassilicos, J. C. 2000 (Ed.) Intermittency in Turbulent Flows. Cambridge University Press.

VAssilicos, J. C.* (Cambridge) Turbulence structure and vortex dynamics: Dynamics of nearsingular vortex; Informal discussions on triad interactions in turbulence: Interscale energy transfer in a compact vortex; Intermittency in turbulent flows and other dynamical systems: Wind tunnel turbulence and intermittency behind a 3-D fractal grid; Future strategies towards understanding and prediction of turbulent systems: intermittency.

VAssilicos, J. C. \& HunT, J. C. R. 1991 Fractal dimensions and spectra of interfaces with application to turbulence. Proc. R. Soc. Lond. A 485, 505-534.

VerzicCO, R. \& JimÉnEZ, J. 1999 On the survival of strong vortex filaments in 'model' turbulence. J. Fluid Mech. 394, 261-279.

VOKE, P.* (Surrey) LES of bypass and separated flow transition.

Voke, P., Sandham, N. D. \& Kleiser, L. (Eds). 1999 Direct and Large-Eddy Simulation III, Proc. Isaac Newton Institute Symposium/ERCOFTAC Workshop, Cambridge, UK, 12-14 May 1999. ERCOFTAC Series, volume 7, Kluwer.

Voth, G. A., Satyanarayan, K. \& Bodenschatz, E. 1998 Lagrangian measurements at large Reynolds numbers. Phys. Fluids 10, 2268-2280.

Vulpiani, A., Biferale, L., Boffetta, G., Celani, A., Cencini, M. \& Vergni, D. 2000 Exit time approach for Lagrangian and Eulerian turbulence. In Intermittency in Turbulent Flows (ed. J. C. Vassilicos). Cambridge University Press.

WAAL, A. VAN DER \& Milton, S. F. 1999 The prediction of extra tropical cyclones and blocking in the UK Met Office global NWP model. Met. Appl. (in press).

WATER, W. VAN DE* (Eindhoven) Angle-dependent structure functions for fully developed turbulence. Warhaft, Z. 2000 Passive scalars in turbulent flows. Ann. Rev. Fluid Mech. 32, 203.

WARHAFT, Z.* (Cornell) 2000 The issue of local isotropy of velocity and scalar turbulent fields. In 
Turbulence Structure and Vortex Dynamics (ed. J. C. R. Hunt \& J. C. Vassilicos). Cambridge University Press.

Water, W. van der, Staicu, A. \& Guegan, M.-C. 2000 Capturing turbulent intermittency. In Intermittency in Turbulent Flows (ed. J. C. Vassilicos). Cambridge University Press.

Wu, X., JACOBS, R. G., Hunt, J. C. R. \& Durbin, P. A. 1999 Simulation of boundary layer transition induced by periodically passing wakes. J. Fluid Mech. 398, 109-153.

Wundrow, D. W. \& Goldstein, M. E. 1999 Can Klebanoff modes produce turbulent spots? submitted to J. Fluid Mech.

Zagarola, M. V. \& Smits, A. J. 1998 Mean-flow scaling of turbulent pipe flow. J. Fluid Mech. 373, 33-79. 